\title{
Dual Weights on Crossed Products by Groupoid Actions
}

\author{
By
}

\author{
Takehiko YAMANOUCHI*
}

\section{§0. Introduction}

In [Y2], we carried out the program of realizing the crossed product by a groupoid action as the left von Neumann algebra of a left Hilbert algebra naturally attached to the given "covariant system". As a consequence, it was shown, as in the case of group actions, that, for each faithful normal positive functional $\varphi$ on the algebra on which the groupoid is acting, there always exists a faithful normal semifinite weight $\widetilde{\varphi}$ on the crossed product, called the dual weight of $\varphi$. (To avoid difficulty, we dealt with a positive functional only, not with a weight). Several expected results were established such as the fact that the modular automorphism group of $\widetilde{\varphi}$ extends that of $\varphi$. It is naturally expected at this stage that this dual weight construction could be done also by exhibiting an operator valued weight of the crossed product to the original algebra, as Haagerup showed in [H4]. The purpose of this paper is to show that this philosophy is indeed the case. The main strategy to achieye our goal can be found in [H4]. However, since we know little about Fourier analysis (or harmonic analysis) on a measured groupoid, we need to provide ourselves with relevant information in this direction. For example, to the best of author's knowledge, no one has ever intensively studied the "Plancherel weight" of a measured groupoid. Thus, naturally, little is known about what functions must be qualified to be called positive definite. Moreover, it should be remarked that the unitary operator $\lambda(\gamma)$ itself, where $\lambda(\cdot)$ is the regular representation of a measured groupoid $\mathscr{G}$, has no meaning in the crossed product $M \times{ }_{\alpha} G$ by an action $\alpha$ of $\mathscr{G}$ (it is not even a member of $M \times_{\alpha} \mathscr{G}$ ), while, in the group case, it is a typical kind of operators that generate the crossed product. This fact makes our argument more difficult than the one in the case of group actions. For example, because of this situation, we need to adopt an approach in $\S 4$ which is different from, but still as interesting as, the ones taken in [H4] or [E\&S].

Communicated by H. Araki, October 7, 1991.

1991 Mathematics Subject Classification: 46L10, 46L55.

* Department of Mathematics and Computer Science, University College of Swansea. Singleton Park, Swansea SA2 8PP United Kingdom. 
Now we would like to describe the plan of the paper. In $\S 1$, we fix a number of notations used throughout this note. We also recall several facts on the groupoid algebra of a measured groupoid. In $\$ 2$, we study the "Plancherel weight" of a measured groupoid in detail and establish several results that will be made use of in the following sections. We should remark that these results themselves are of great interest. They will be treated in more detail elsewhere. The section 3 is concerned with construction of an operator valued weight $T_{\alpha}$ of the crossed product by a groupoid action $\alpha$ to the algebra on which the groupoid is acting. The approach taken here is related to [E\&S]. Roughly speaking, the idea of how to construct $T_{\alpha}$ is to "slice" the dual coaction $\widehat{\alpha}$ by the Plancherel weight $\varphi_{g}$ of the groupoid $\mathscr{G}_{:} T_{\alpha}=\left(\iota *_{\mu} \varphi_{g}\right) \circ \widehat{\alpha}$. In $\S 4$, we prove that the dual weight obtained through the method of [Y2] can be captured by composing the originally given functional with the operator valued weight $T_{\alpha}$. As we mentioned above, the approach taken here is new. The key observation is Proposition 4.1, which itself is of interest. Note that, in [Y2], we dealt with only the dual weights of faithful positive functionals. However, the result of this paper suggests that it should be possible to extend the construction of [Y2] from positive functionals to weights.

\section{§1. Notations}

In this section, we fix notations used in the following sections. We shall also recall several facts on a left Hilbert algebra constructed from a measured groupoid.

Throughout this paper, we fix a standard Borel groupoid $\mathscr{G}$ once and for all. We assume that all relevant maps and sets that are related to the groupoid structure of $\mathscr{G}$ are Borel. We denote the source (resp. the range) of an element $\gamma$ of the groupoid by $s(\gamma)$ (resp. $r(\gamma)$ ). The unit space of $\mathscr{G}$, which is the image of the groupoid under the source (or the range) map, is denoted by $X$. For every $x \in X, \mathscr{G}^{x}$ (resp. $\mathscr{G}_{x}$ ) designates the inverse image of the range (resp. the source) map $r^{-1}(x)$ (resp. $s^{-1}(x)$ ). By a measured groupoid $\mathscr{G}$, we mean that $\mathscr{G}$ admits a faithful proper transverse function $\left\{\lambda^{x}\right\}_{x \in X}$ and a transverse measure $\Lambda$ with a module $\delta$. If a system $\left(\mathscr{G},\left\{\lambda^{x}\right\}, \Lambda, \delta\right)$ is measured, then there canonically exists a $\sigma$-finite measure $\mu$ on the unit space $X$ (see [C1]). Using the measure $\mu$, we can

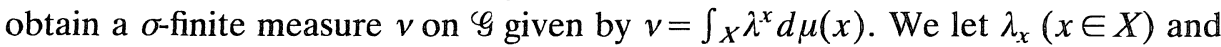
$v^{-1}$ be the measures on $\mathscr{G}$ defined by

$$
\lambda_{x}(f)=\lambda^{x}(\widetilde{f}), \quad v^{-1}(f)=v(\widetilde{f})
$$

for any positive Borel function $f$ on $\mathscr{G}$, where $\widetilde{f}(\gamma)=f\left(\gamma^{-1}\right)(\gamma \in \mathscr{G})$. It is known that $v$ is equivalent to $v^{-1}$, and that the Radon-Nikodym derivative $d v / d v^{-1}$ is the module $\delta$. Let $\mathscr{Z}$ be the abelian von Neumann algebra $L^{\infty}(X, \mu)$. Then the Hilbert space $L^{2}(\mathscr{G}, v)$ admits two natural $\mathscr{L}$-module structures; one is derived 
from the representation $h \in \mathscr{L} \mapsto M(h \circ r)$, and the other comes from the representation $h \in \mathscr{E} \mapsto M(h \circ s)$, where $M(f)$ denotes the multiplication operator by $f \in L^{\infty}(G, v)$. We write $\mathscr{L} L^{2}(\mathscr{G}, v)$ (resp. $\left.L^{2}(G, v)_{\mathscr{Z}}\right)$ for $L^{2}(\mathscr{G}, v)$ when the former (resp. the latter) $\mathscr{L}$-module structure is specifically considered on $L^{2}(\mathscr{G}, v)$. We define $D\left(\mathscr{x} L^{2}(G, v), \mu\right)$ to be the set of all $\mu$-bounded vectors in $L^{2}(\mathscr{G}, v)$ relative to the action $h \mapsto M(h \circ r)$ of $\mathscr{Z}$ with the faithful normal semifinite trace $\mu$. We refer readers to [C2] for the definition of a $\varphi$-bounded vector. We also define $D\left(L^{2}(\mathscr{G}, v)_{\mathscr{x}}, \mu\right)$ similarly for the other action. By Lemma 2.1 of [Y1], $\xi$ is in $D\left({ }_{x} L^{2}(G, v), \mu\right)$ if and only if $\lambda\left(|\xi|^{2}\right) \in L^{x}(X, \mu)$, where $\lambda(f)$ is a function on $X$ defined by $\lambda(f)(x)=\int f(\gamma) d \lambda^{x}(\gamma)$ for any Borel function $f$ on $\mathscr{G}$. On the other hand, $\xi$ is in $D\left(L^{2}(G, v)_{\mathscr{Q}}, \mu\right)$ precisely when $\lambda^{\prime}\left(|\xi|^{2}\right) \in L^{\infty}(X, \mu)$, where $\lambda^{\prime}(f)$ is a function on $X$ given by $\lambda^{\prime}(f)(x)=\int f(\gamma) d \lambda_{x}^{\prime}(\gamma)$ with $d \lambda_{x}^{\prime}=\delta d \lambda_{x}$.

In [Y2], we associated a left Hilbert algebra $\mathfrak{B}_{I}$ with a given measured groupoid $\left(\mathscr{G},\left\{\lambda^{x}\right\}, \Lambda, \delta\right)$. It was defined to be the set of all functions $f$ in $D\left({ }_{\mathscr{L}} L^{2}(G, v), \mu\right) \cap D\left(L^{2}(\mathscr{G}, v)_{\mathscr{L}}, \mu\right)$ such that $f$ is $\delta$-bounded and $\|f\|_{I}<\infty$, where $\|\cdot\|_{I}$ is given by $\|g\|_{I}=\max \left\{\|\lambda(|g|)\|_{\infty},\left\|\lambda^{\prime}(|g|)\right\|_{\infty}\right\}$. Refer to [Ha] for the definition of $\delta$-boundedness. It is shown in $\S 1$ of [Y2] that $\mathfrak{B}_{I}$ is a left Hilbert algbera (in fact, a Tomita algebra) in $L^{2}(G, v)$ with the usual convolution $*$ as product and $f^{\#}(\gamma)=\delta(\gamma)^{-1} \overline{f\left(\gamma^{-1}\right)}$ as \#-operation. The modular operator $\Delta$ and the modular conjugation $J$ are given by

$$
\{\Delta f\}(\gamma)=\delta(\gamma) f(\gamma) \quad\left(f \in \mathfrak{B}_{I}\right) \quad\{J f\}(\gamma)=\delta(\gamma)^{-1 / 2} \overline{f\left(\gamma^{-1}\right)} .
$$

The b-operation is $f^{b}(\gamma)=\overline{f\left(\gamma^{-1}\right)}$. We denote by $\pi_{\ell}$ (resp. $\pi_{r}$ ) the left (resp. the right) multiplication of the Tomita algebra $\mathfrak{B}_{I}$. Namely, $\pi_{\ell}(f) g=f * g=\pi_{r}(g) f$ for $f, g \in \mathfrak{B}_{I}$. We may extend $\pi_{\ell}$ (resp. $\pi_{r}$ ) to the left bounded vectors (resp. the right bounded vectors). The left von Neumann algebra of $\mathfrak{B}_{I}$ is called the groupoid von Neumann algebra $\mathscr{R}(\mathscr{G})$ derived from the measured groupoid $(\mathscr{G}$, $\left.\left\{\lambda^{x}\right\}, \Lambda, \delta\right)$. It is known that this von Neumann algebra is equipped with a $\mathscr{Z}$-module structure via the $*$-isomorphism $h \in \mathscr{E} \mapsto M(h \circ r)$. We sometimes write $\mathscr{E}_{R}$ for the image of $\mathscr{E}$ under this isomorphism, but we identify $\mathscr{E}_{R}$ with $\mathscr{E}$ most of the times. The image $\mathscr{E}_{S}$ of $\mathscr{L}$ under the other isomorphism $h \in \mathscr{L} \mapsto M(h \circ s)$ is contained in the commutant of $\mathscr{R}(\mathscr{G})$. Let $\varphi_{\mathscr{G}}$ denote the faithful normal semifinite weight on $\mathscr{R}(\mathscr{G})$ associated with $\mathfrak{B}_{I}$. We call $\varphi_{\mathscr{G}}$ the Plancherel weight of $\mathscr{G}$. Besides the Tomita algebra $\mathfrak{B}_{I}$, we would like to introduce a subset $\mathfrak{B}_{I}^{x}$ of $\mathfrak{B}_{I}$ that is defined by $\mathfrak{B}_{I}^{\infty}=\mathfrak{B}_{I} \cap L^{\infty}(\mathscr{G}, v)$. The set $\mathfrak{B}_{I}^{\infty}$ is contained in all the relevant spaces such as $L^{2}(\mathscr{G}, v), L^{\infty}(\mathscr{G}, v), D\left({ }_{\mathscr{L}} L^{2}(\mathscr{G}, v), \mu\right)$ etc., and yet it is "sufficiently large", as you will see in a moment. So $\mathfrak{B}_{I}^{\infty}$ can be regarded as a "good" substitute in the measure-theoretic setting for $\mathscr{K}(\mathscr{G})$, the set of all continuous functions with compact support, in the topological setting. $\mathfrak{B}_{I}^{\infty}$ turns out to be a \#-subalgebra of $\mathfrak{B}_{I}$. Indeed, if $f, g \in \mathfrak{B}_{I}^{\infty}$, then we have that $\|f * g\|_{\infty} \leq$ $\left\|\lambda\left(|f|^{2}\right)\right\|_{\infty}^{1 / 2}\left\|\lambda\left(|g|^{2}\right)\right\|_{\infty}^{1 / 2}$ by Schwarz inequality; so $f * g \in \mathfrak{B}_{I}^{x}$. A vector $f$ is $\delta_{a^{-}}$ bounded if and only if $f^{\#}$ is $\delta_{a}$-bounded; so $f \in \mathfrak{B}_{I}^{\infty} \Leftrightarrow f^{\#} \in \mathfrak{B}_{I}^{\infty}$. Moreover, by the 
same argument as in Lemma 1.7 of [Y2], we may prove that $\mathfrak{B}_{I}^{\infty}$ is a left Hilbert algebra (in fact, a Tomita algebra) equivalent to $\mathfrak{B}_{I}\left(\right.$ namely, $\left.\mathfrak{B}_{I}^{\infty}\right){ }^{\prime \prime}=\mathfrak{B}_{I}^{\prime \prime}$ ). Accordingly, the left von Neumann algebra of $\mathfrak{B}_{I}^{\infty}$ is $\mathscr{R}(\mathscr{\varphi})$. It should be remarked that all the results established in [Y2] involving the left Hilbert algebra $\mathfrak{B}_{I}$ still hold valid even if we substitute $\mathfrak{B}_{I}^{\infty}$ for $\mathfrak{B}_{I}$ in the proofs or discussion. For example, in the definition of $\mathscr{F}_{\varphi}(\mathcal{M})$ (see $\$ 2$ of [Y2]), the condition (F1) can be replaced as follows: (F1)' $f_{a} \in L^{\infty}(\mathscr{G}, v),\left\|f_{a}\right\|_{I}<\infty$ and $\lambda\left(f_{a}^{2}\right), \lambda^{\prime}\left(f_{a}^{2}\right) \in L^{\infty}(X, \mu)$, where $f_{a}(\gamma)=\|a(\gamma)\|$. Let us denote by $\mathscr{F}_{\varphi}^{\infty}(\mathcal{M})$ (or simply by $\mathscr{F}_{\varphi}^{\infty}$ ) the set of all such $a$ 's. Then all the arguments in \$2 of [Y2] work even for $\mathscr{F}_{\varphi}^{\infty}$. Moreover, the crossed product $M \times_{\alpha} \mathscr{G}$ by a groupoid action $\alpha$ is generated by elements of the forms $a \otimes_{\mathscr{L}} 1(a \in \mathcal{M})$ and $(u \otimes \lambda)(f)\left(f \in \mathfrak{B}_{I}^{\infty}\right)$ (see Theorem 2.14 of [Y2]).

For a weight $\chi$ on a von Neumann algebra $\mathscr{P}$, we use the conventional notations as follows:

$$
\begin{aligned}
\mathrm{p}_{\chi} & =\left\{a \in \mathscr{P}_{+}: \chi(a)<\infty\right\} \\
\mathrm{n}_{\chi} & =\left\{a \in \mathscr{P}: \chi\left(a^{+} a\right)<\infty\right\} \\
\mathrm{m}_{\chi} & =\mathrm{n}_{\chi}^{+} \mathrm{n}_{\chi}=\operatorname{span}\left\{x^{+} y: x, y \in \mathrm{n}_{\chi}\right\} .
\end{aligned}
$$

For an operator valued weight $E$ from $\mathscr{P}$ onto a von Neumann subalgebra 2 , we put

$$
\begin{aligned}
\mathrm{p}_{E} & =\left\{a \in \mathscr{P}_{+}: E(a) \in \mathscr{Q}_{+}\right\} \\
\mathrm{n}_{E} & =\left\{a \in \mathscr{P}: a^{*} a \in \mathrm{p}_{E}\right\} \\
\mathrm{m}_{E} & =\mathrm{n}_{E}^{+} \mathrm{n}_{E}=\operatorname{span}\left\{x^{+} y: x, y \in \mathrm{n}_{E}\right\}
\end{aligned}
$$

Important results on operator valued weights can be found in the literatures [H2] and [H3].

Finally, we refer readers to [S1] for fundamental facts on relative tensor products of Hilbert spaces and von Neumann algebras over an abelian von Neumann algebra.

\section{§2. The Plancherel Weight $\varphi_{G}$}

In this section, we investigate the Plancherel weight $\varphi_{g}$ in great detail. Then we establish a result (Proposition 2.7) that will be used in the next section. In the course of discussion, we introduce a set, denoted by $\mathscr{P}(\mathscr{G})$, which is connected with "positive definite functions". This set will play an important role in the following sections.

Let $\mathscr{P}$ and $\mathscr{Q}$ be von Neumann algebras acting on Hilbert spaces $\mathscr{H}_{1}$ and $\mathscr{H}_{2}$, respectively. We assume that these algebras are both $\mathscr{L}$-modules. We form the $\left(\mathscr{Z}\right.$-)fiber product $\mathscr{P} *_{\mathscr{L}} \mathscr{Q}$ on the relative tensor product $\mathscr{H}_{1} \otimes_{\mu} \mathscr{H}_{2}$. For normal positive linear functionals $\omega_{1}$ and $\omega_{2}$ on $\mathscr{P}$ and 2 , respectively, satisfying 


$$
\mu\left(\frac{d \omega_{1}}{d \mu} \cdot \frac{d \omega_{2}}{d \mu}\right)<\infty,
$$

a normal positive function $\omega_{1} *_{\mu} \omega_{2}$, called the $\mathscr{L}$-product of $\omega_{1}$ and $\omega_{2}$, on $\mathscr{P} *_{\mathscr{L}} \mathscr{2}$ was defined in Proposition III.4 of [S1], where $d \omega_{i} / d \mu(i=1,2)$ are the RadonNikodym derivatives of the restrictions of $\omega_{i}$ 's to $\mathscr{E}$ with respect to $\mu$. If $\omega_{i}$ 's are vector functionals of the form $\omega_{1}=\omega_{\xi_{1}}\left(\xi_{i} \in \mathscr{H}_{i}(i=1,2)\right)$ with $\xi_{i} \in D\left(\mathscr{H}_{i}, \mu\right)=$ the set of $\mu$-bounded vectors in $\mathscr{H}_{i}$, then the condition (*) is automatically satisfied and $\omega_{1} *_{\mu} \omega_{2}$ turns out be the vector functional $\omega_{\xi_{1} \otimes_{11} \xi_{2}}$. This fact will be often used hereafter in our discussion.

Next we introduce a concept of a $\mathscr{L}$-weight (Definition III.10 of [S1]). A weight $\phi$ on a von Neumann algbera $\mathfrak{Y}$ with a $\mathscr{L}$-module structure is called a $\mathscr{L}$-weight if $\phi\left(u x u^{4}\right)=\phi(x)$ for any $x \in \mathfrak{P}_{+}$and any unitary $u \in \mathscr{L}$. Let $\mathscr{P}$ and $\mathscr{Q}$ be as above. Suppose that $\mathscr{P}$ admits a $\mathscr{L}$-weight $\phi$. We define

$$
\Omega_{\phi}=\left\{\omega \in \mathscr{P}_{+}^{+}: \omega(x) \leq \phi(x) \text { for all } x \in \mathscr{P}_{+}\right\} .
$$

It is well-known that $\phi(x)=\sup \left\{\omega(x): \omega \in \Omega_{\phi}\right\}$ for any $x \in \mathscr{P}_{+}$. If $\chi$ is a normal positive functional on 2 with $d \chi / d \mu$ bounded, then we put

$$
\phi *_{\mu} \chi=\sup _{\omega \in \Omega_{\phi}} \omega *_{\mu} \chi .
$$

If $\chi$ is in $2_{+}^{+}$with $d \chi / d \mu$ not necessarily bounded, then we choose a faithful normal semifinite trace $\tau$ on $\mathscr{E}$ with $d \chi / d \tau$ bounded, and set

$$
\phi *_{\mu} \chi=\phi *_{\tau} \chi\left(\frac{d \tau}{d \mu} .\right) .
$$

It is shown in [S1] that this definition is independent of the choice of $\tau$ with $d \chi / d \tau$ bounded. In any case, the weight $\phi *_{\mu} \chi$ turns out to be a normal weight on $\mathscr{P} * \mathscr{2}$. These are the notions necessary for our later argument.

Now we begin to make a close investigation on the Plancherel weight of a measured groupoid.

Lemma 2.1. The algebra $\mathscr{Z}_{R}$ is contained in the centralizer of $\varphi \mathrm{g}$.

Proof. Recall that the modular operator $\Delta$ of $B_{I}$ is defined by

$$
\{\Delta g\}(\gamma)=\delta(\gamma) g(\gamma) \quad\left(g \in \mathfrak{B}_{l}\right) .
$$

Then it follows that $\Delta^{i t} M(h \circ r) \Delta^{-i t}=M(h \circ r)$ for any $h \in \mathscr{Z}$ and $t \in \mathbf{R}$. Q.E.D.

Lemma 2.2. There exists a unique faithful normal semifinite operator valued weight $T$ from $\mathscr{R}(\mathscr{G})$ onto $\mathscr{W}_{R}$ such that $\varphi_{g}=\mu \circ T$.

Proof. By the previous Lemma, we have $\sigma_{t}^{\varphi_{\mathfrak{s}}}=i d=\sigma_{t}^{\mu}$ on $\mathscr{L}\left(=\mathscr{L}_{R}\right)$, where 
$\sigma_{t}^{\chi}$ denotes the modular automorphism of a faithful normal semifinite weight $\chi$ on a von Neumann algebra. The assertion now follows from Theorem 5.1 of [H3].

Q.E.D.

Lemma 2.3. The weight $\varphi_{\mathrm{g}}$ is a $\mathscr{L}$-weight on $\mathscr{R}(\mathscr{G})$.

Proof. Let $u \in \mathscr{L}$ be a unitary. Then, due to the preceding Lemma, we have

$$
\begin{aligned}
\varphi_{g}\left(u a u^{+}\right) & =\mu \circ T\left(\text { uau }^{+}\right) \\
& =\mu\left(u T(a) u^{+}\right) \\
& =\mu \circ T(a)=\varphi_{g}(a)
\end{aligned}
$$

for any $a \in \mathscr{R}(\mathscr{G})_{+}$.

Q.E.D.

We define a set $\mathscr{P}_{0}(\mathscr{G})$ to be the set of vectors $h \in L^{2}(\mathscr{G}, v)$ satisfying

$$
\int \overline{\left(h * h^{b}\right)(\gamma)}\left(f^{\#} * f\right)(\gamma) \leq \int|f(\gamma)|^{2} d v(\gamma)
$$

for all Borel functions $f \in \mathfrak{B}_{I}$. Several remarks on $\mathscr{P}_{0}(\mathscr{G})$ are in order. Firstly, Fubini's theorem tells us that the left-hand side of (2.4) equals $\left\|\pi_{\ell}(f) h\right\|^{2}$. Meanwhile, the right-hand side is equal to $\|f\|^{2}$, which, by definition, equals $\varphi_{\mathscr{G}}\left(\pi_{\ell}(f)^{4} \pi_{\ell}(f)\right)$. Consequently, inequality (2.4) is equivalent to $\left\|\pi_{\ell}(f) h\right\|^{2} \leq$ $\|f\|^{2}$. Thus the set $\mathscr{P}_{0}(\mathscr{G})$ coincides with the set of all right bounded vectors $h$ with $\left\|\pi_{r}(h)\right\| \leq 1$. Secondly, we assert that

$$
\Omega_{\varphi_{\text {ig }}}=\left\{\omega_{h}: h \in \mathscr{P}_{0}(\mathscr{G})\right\} .
$$

In fact, if $h \in \mathscr{P}_{0}(\mathscr{G})$, them, for any left bounded vector $f$, we have

$$
\begin{aligned}
\omega_{h}\left(\pi_{\ell}(f)^{+} \pi_{\ell}(f)\right) & =\left\|\pi_{\ell}(f) h\right\|^{2} \\
& =\left\|\pi_{r}(h) f\right\|^{2} \\
& \leq\|f\|^{2}=\varphi_{g}\left(\pi_{\ell}(f)^{+} \pi_{\ell}(f)\right) .
\end{aligned}
$$

It follows that $\omega_{h}(a) \leq \varphi_{g}(a)$ for any $a \in \mathscr{R}(\mathscr{G})_{+}$. Conversely, suppose that $\omega \in \Omega_{\varphi_{\zeta} \text {. Since }}\left\{\mathscr{R}(\mathscr{G}), L^{2}(\mathscr{G}, v)\right\}$ is a standard representation, there exists a vector $h \in L^{2}(\mathscr{G}, v)$ with $\omega=\omega_{h}$. Then, for any $f \in \mathcal{B}_{I}$, we compute

$$
\begin{aligned}
\left\|\pi_{\ell}(f) h\right\|^{2} & =\omega\left(\pi_{\ell}(f)^{*} \pi_{\ell}(f)\right) \\
& \leq \varphi_{g}\left(\pi_{\ell}(f)^{*} \pi_{\ell}(f)\right)=\|f\|^{2} .
\end{aligned}
$$

Thus $h \in \mathscr{P}_{0}(\mathscr{G})$. Therefore (2.5) follows. From (2.5), it results that, for each $a \in \mathscr{R}(\mathscr{G})_{+}$, we have

$$
\varphi_{\mathscr{G}}(a)=\sup \left\{\omega_{h}(a): h \in \mathscr{P}_{0}(\mathscr{G})\right\}
$$


However, a more powerful identity holds true as you shall see below. Before we state the identity, we introduce a new set. We put

$$
\mathscr{P}(\mathscr{G})=\mathscr{P}_{0}(\mathscr{G}) \cap D\left(\mathscr{L} L^{2}(\mathscr{G}, v), \mu\right) \text {. }
$$

This set will play a central role in the discussion that follows.

Remark 2.6. From the above remarks on $\mathscr{P}_{0}(\mathscr{G})$, it follows that a vector $h$ in $L^{2}(\mathscr{G}, v)$ belongs to $\mathscr{P}(\mathscr{G})$ if and only if $h$ is a right bounded vector in $D\left({ }_{\mathscr{L}} L^{2}(\mathscr{G}, v), \mu\right)$ with $\left\|\pi_{r}(h)\right\| \leq 1$.

Proposition 2.7. For every $a \in \mathscr{R}(\mathscr{G})_{+}$, we have

$$
\varphi_{g}(a)=\sup \left\{\omega_{h}(a): h \in \mathscr{P}(\mathscr{G})\right\} \text {. }
$$

Proof. Due to (2.5), it suffices to show that the right-hand side of the assertion majorizes the other. Before we proceed further, we make one observation. Let $f \in \mathscr{B}_{I}$ and $g \in \mathscr{P}_{0}(\mathscr{G})$. Since $f$ is in $D\left(\mathscr{L} L^{2}(\mathscr{G}, v), \mu\right)$, there is a positive number $C$ such that $\|M(k \circ r) f\|^{2} \leq C \int|k(x)|^{2} d \mu(x)\left(k \in \mathrm{n}_{\mu}\right)$. Then, for any $k \in \mathrm{n}_{\mu}$, we have

$$
\begin{aligned}
\left\|M(k \circ r) \pi_{\ell}(f) g\right\|^{2} & =\left\|M(k \circ r) \pi_{r}(g) f\right\|^{2} \\
& =\left\|\pi_{r}(g) M(k \circ r) f\right\|^{2} \\
& \leq\|M(k \circ r) f\|^{2} \\
& \leq C \int|k(x)|^{2} d \mu(x) .
\end{aligned}
$$

The second equality is guaranteed by the fact that $M(k \circ r)$ commutes with $\pi_{r}(g)$. The third inequality is due to the remark on $\mathscr{P}_{0}(\mathscr{G})$. The computation means that the vector $\pi_{\ell}(f) g$ belongs to $D\left({ }_{\mathscr{L}} L^{2}(\mathscr{G}, v), \mu\right)$. Since $\mathfrak{B}_{I}$ is a Tomita algebra, every element in $\mathfrak{B}_{I}$ is right bounded; so the vector $\pi_{\ell}(f) g=f * g$ is also right bounded. Moreover, we have $\left\|\pi_{r}(f * g)\right\|=\left\|\pi_{r}(g) \pi_{r}(f)\right\| \leq\left\|\pi_{r}(f)\right\|$. It follows from Remark 2.6 that the vector $\pi_{\ell}(f) g$ lies in $\mathscr{P}(\mathscr{G})$ if $g \in \mathscr{P}_{0}(\mathscr{G})$ and $f \in \mathfrak{B}_{I}$ with $\left\|\pi_{r}(f)\right\| \leq 1$.

Now we move on to the proof of the assertion. Let $a \in \mathscr{R}(\mathscr{G})_{+}$and $g \in \mathscr{P}_{0}(\mathscr{G})$. First we assume that $a^{1 / 2} \in \mathrm{n}_{\varphi_{c},}$. Namely, $a^{1 / 2}$ is of the form $a^{1 / 2}=\pi_{\ell}(p)$ for some left bounded vector $p$. Since $\pi_{r}\left(\mathfrak{B}_{I}\right)$ is a $\sigma$-strongly dense $*$-subalgebra of the commutant $\mathscr{R}(\mathscr{G})^{\prime}$, it follows from Kaplansky's density theorem that there exists a net $\left\{f_{i}\right\}$ in $\mathfrak{B}_{I}$ such that $\left\|\pi_{r}\left(f_{i}\right)\right\| \leq 1$ and the net $\left\{\pi_{r}\left(f_{i}\right)\right\}$ converges $\sigma$-strongly to 1 . Due to the first paragraph, vectors $\pi_{\ell}\left(f_{i}\right) g$ are in $\mathscr{P}(\mathscr{G})$. Moreover,

$$
\begin{aligned}
\lim _{i} \omega_{\pi_{\ell}\left(f_{t}\right) g}(a) & =\lim _{i}\left\|\pi_{\ell}(p) \pi_{\ell}\left(f_{i}\right) g\right\|^{2} \\
& =\lim _{i}\left\|\pi_{\ell}(p) \pi_{r}(g) f_{i}\right\|^{2} \\
& =\lim _{i}\left\|\pi_{r}(g) \pi_{\ell}(p) f_{i}\right\|^{2} \\
& =\lim _{i}\left\|\pi_{r}(g) \pi_{r}\left(f_{i}\right) p\right\|^{2}
\end{aligned}
$$




$$
\begin{aligned}
& =\left\|\pi_{r}(g) p\right\|^{2} \\
& =\left\|\pi_{\ell}(p) g\right\|^{2}=\omega_{g}(a) .
\end{aligned}
$$

This implies

$$
\omega_{g}(a) \leq \sup \left\{\omega_{h}(a): h \in \mathscr{P}(\mathscr{G})\right\} .
$$

Thus, by (2.5), we have

$$
\varphi_{\mathscr{G}}(a) \leq \sup \left\{\omega_{h}(a): h \in \mathscr{P}(\mathscr{G})\right\} .
$$

Next we would like to show that the quantity $\sup \left\{\omega_{h}(a): h \in \mathscr{P}(\mathscr{G})\right\}$ is infinite if $a^{1 / 2} \notin \mathrm{n}_{\varphi_{\varphi}}$. Suppose that it were finite when $a^{1 / 2} \notin \mathrm{n}_{\varphi_{\varphi},}$. Without any loss of generality, we may assume that $\sup \left\{\omega_{h}(a): h \in \mathscr{P}(\mathscr{G})\right\}=1$. Since $\left\{h \in \mathscr{B}_{I}\right.$ : $\left.\left\|\pi_{r}(h)\right\| \leq 1\right\} \subseteq \mathscr{P}(\mathscr{G})$, it results that

$$
\left\|a^{1 / 2} h\right\| \leq\left\|\pi_{r}(h)\right\|
$$

for any $h \in \mathfrak{B}_{\digamma}$. By this inequality, the equation

$$
\zeta\left(\pi_{r}(h)\right)=a^{1 / 2} h
$$

defines a continuous linear map $\zeta$ from $\pi_{r}\left(\mathfrak{B}_{I}\right)$ into $L^{2}(\varphi, v)$. So we may extend $\zeta$ to a bounded linear map, denoted by $\zeta$ again, from the uniform closure $A_{r}$ of $\pi_{r}\left(\mathfrak{B}_{I}\right)$, which is a (nondegenerate separable) $C^{+}$-algebra. For any $h, k \in \mathfrak{B}_{I}$, we have

$$
\begin{aligned}
\zeta\left(\pi_{r}(h) \pi_{r}(k)\right) & =\zeta\left(\pi_{r}(k * h)\right) \\
& =a^{1 / 2}(k * h) \\
& =a^{1 / 2} \pi_{r}(h) k \\
& =\pi_{r}(h) a^{1 / 2} k \\
& =\pi_{r}(h) \zeta\left(\pi_{r}(k)\right) .
\end{aligned}
$$

Thus continuity of $\zeta$ yields

$$
\zeta\left(b_{1} b_{2}\right)=b_{1} \zeta\left(b_{2}\right)
$$

for any $b_{1}, b_{2} \in A_{r}$. Let $\left\{u_{n}\right\}_{n \geq 1}$ be a countable approximate identity for $A_{r}$. Then we have that $\left\|\zeta\left(u_{n}\right)\right\| \leq 1$ for any $n \geq 1$. Since the unit ball of $L^{2}(\mathscr{G}, v)$ is weakly compact, there exists a subsequence of $\left\{\zeta\left(u_{n}\right)\right\}$ that converges weakly to an element $p_{0}$ in $L^{2}(\xi, v)$. Without loss of generality, we can assume that $\left\{\zeta\left(u_{n}\right)\right\}$ itself converges weakly to $p_{0}$. Then, for any $k, k_{1} \in \mathfrak{B}_{l}$, we have

$$
\begin{aligned}
\left(\pi_{r}(k) p_{0} \mid k_{1}\right) & =\left(p_{0} \mid \pi_{r}(k)^{+} k_{1}\right) \\
& =\lim _{n \rightarrow \infty}\left(\zeta\left(u_{n}\right) \mid \pi_{r}(k)^{+} k_{1}\right) \\
& =\lim _{n \rightarrow \infty}\left(\zeta\left(\pi_{r}(k) u_{n}\right) \mid k_{1}\right)
\end{aligned}
$$




$$
\begin{aligned}
& =\left(\zeta\left(\pi_{r}(k)\right) \mid k_{1}\right) \\
& =\left(a^{1 / 2} k \mid k_{1}\right) .
\end{aligned}
$$

Thus we obtain $\pi_{r}(k) p_{0}=a^{1 / 2} k\left(k \in \mathfrak{B}_{I}\right)$. It follows that $p_{0}$ is a left bounded vector with $\pi_{\ell}\left(p_{0}\right)=a^{1 / 2}$, which is a contradiction. Consequently, the quantity $\sup \left\{\omega_{h}(a): h \in \mathscr{P}(\mathscr{G})\right\}$ is infinite if $a^{1 / 2} \notin \mathrm{n}_{\varphi_{\xi}}$. Since $\varphi_{g}(a)=\infty$ if $a^{1 / 2} \notin \mathbf{n}_{\varphi \cdot g}$, we get the desired identity.

Q.E.D.

As a consequence of Proposition 2.7, we obtain the equality:

$$
\sup _{h \in \mathscr{P}(\mathcal{G})} \int\left(f^{\#} * f\right)(\gamma) \overline{\left(h * h^{b}\right)(\gamma)} d v(\gamma)=\int|f(\gamma)|^{2} d v(\gamma)
$$

for any left bounded vector $f$ in $L^{2}(G, v)$. However, we can prove a much stronger identity as below.

Proposition 2.8. Suppose that $f$ is a Borel function on $G$. Then we have

$$
\sup _{h \in \mathscr{P}(\mathscr{G})} \int\left(f^{\#} * f\right)(\gamma) \overline{\left(h * h^{b}\right)(\gamma)} d v(\gamma)=\int|f(\gamma)|^{2} d v(\gamma) \text {. }
$$

The above quantity may be infinite.

Proof. First we assume that $f \in L^{2}(G, v)$. Then, as we noted before, the desired identity can be written as

$$
\sup _{h \in \mathscr{P}(\mathscr{G})}\left\|\pi_{r}(h) f\right\|^{2}=\|f\|^{2} .
$$

Since $\left\|\pi_{r}(h)\right\| \leq 1$ for any $h \in \mathscr{P}(\mathscr{G})$ by Remark 2.6 , it follows that $\sup _{h \in \mathscr{P}(\mathscr{G})}$ $\left\|\pi_{r}(h) f\right\|^{2} \leq\|f\|^{2}$. Recall that $\left\{h \in \mathfrak{B}_{I}:\left\|\pi_{r}(h)\right\| \leq 1\right\}$ is a subset of $\mathscr{P}(\mathscr{G})$. Thus, by Kaplansky's density theorem, there exists a net $\left\{h_{i}\right\}$ in $\mathscr{P}(\mathscr{G})$ such that $\left\{\pi_{r}\left(h_{i}\right)\right\}$ converges $\sigma$-strongly ${ }^{+}$to 1 . Hence

$$
\|f\|^{2}=\lim _{i}\left\|\pi_{r}\left(h_{i}\right) f\right\|^{2} \leq \sup _{h \in \mathscr{P}(\mathscr{G})}\left\|\pi_{r}(h) f\right\|^{2} .
$$

Therefore we obtain the assertion.

Now we assume that $f$ is not in $L^{2}(G, v)$. It suffies to show that the left-hand side of the desired identity is infinite, but we suppose that it were bounded by a positive number $M$. Then, by Fubini's theorem, we have that $f * h \in L^{2}(\mathscr{G}, v)$ for any $h \in \mathscr{P}(\mathscr{G})$. Since $\left\{h \in \mathfrak{B}_{I}:\left\|\pi_{r}(h)\right\| \leq 1\right\} \subseteq \mathscr{P}(\mathscr{G})$, it follows that, for any $h \in \mathfrak{B}_{I}, f * h \in L^{2}(\mathscr{G}, v)$ and

$$
\|f * h\| \leq M^{1 / 2}\left\|\pi_{r}(h)\right\| .
$$

By arguing like the previous Proposition, we may obtain a bounded linear map $\xi$ from the $C^{+}$-algebra $A_{r}$, the uniform closure of $\pi_{r}\left(\mathfrak{B}_{I}\right)$, into $L^{2}(\mathscr{G}, v)$ characterized by the equation 


$$
\xi\left(\pi_{r}(h)\right)=f * h .
$$

We observe that, for any $h, k \in \mathfrak{B}_{I}$, we have

$$
\begin{aligned}
\xi\left(\pi_{r}(k) \pi_{r}(h)\right) & =\xi\left(\pi_{r}(h * k)\right)=f * h * k \\
& =\pi_{r}(k)(f * h)=\pi_{r}(k) \xi\left(\pi_{r}(h)\right) .
\end{aligned}
$$

Thanks to the continuity of $\xi$, we deduce that

$$
\xi(a b)=a \xi(b)
$$

for any $a, b \in A_{r}$. Let us fix an element $k \in \mathfrak{B}_{I}$. Then we define a linear functional $\varphi_{k}$ on $A_{r}$ by

$$
\varphi_{k}(a)=(\xi(a) \mid k) \quad\left(a \in A_{r}\right),
$$

which is bounded, because $\xi$ is continuous. Again, by arguing like Proposition 2.7 , we can assume that the sequence $\left\{\xi\left(u_{n}\right)\right\}_{n \geq 1}$ converges weakly to an element $g \in L^{2}(\mathscr{G}, v)$ if $\left\{u_{n}\right\}$ is a countable approximate identity for $A_{r}$. Then, for any $a \in A_{r}$, we have

$$
\begin{aligned}
\varphi_{k}(a) & =\lim _{n \rightarrow \infty} \varphi_{k}\left(a u_{n}\right)=\lim _{n \rightarrow \infty}\left(\xi\left(a u_{n}\right) \mid k\right) \\
& =\lim _{n \rightarrow \infty}\left(a \xi\left(u_{n}\right) \mid k\right)=\lim _{n \rightarrow \infty}\left(\xi\left(u_{n}\right) \mid a^{+} k\right) \\
& =\left(g \mid a^{*} k\right)=(a g \mid k) .
\end{aligned}
$$

This shows that

$$
(\xi(a) \mid k)=(a g \mid k)
$$

for any $a \in A_{r}$. Upon replacing $a$ by $\pi_{r}(h)\left(h \in \mathfrak{B}_{I}\right)$, we get

$$
(f * h \mid k)=(g * h \mid k)
$$

for any $h \in \mathfrak{B}_{I}$. Let $h, k \in \mathfrak{B}_{I}$. Then, by Fubini's theorem,

$$
\int f(\gamma) \overline{\left(k * h^{b}\right)(\gamma)} d v(\gamma)=(f * h \mid k)=(g * h \mid k)=\left(g \mid k * h^{b}\right) .
$$

This shows that

$$
\int f(\gamma) \overline{p(\gamma)} d v(\gamma)=(g \mid p)
$$

for every $p \in \mathfrak{B}_{I}^{2}$. Since the unit ball of $\mathfrak{B}_{I}^{2}$ is dense in that of $L^{2}(\mathscr{G}, v)$, we have

$$
\begin{aligned}
\|f\| & =\sup \left\{\left|\int f(\gamma) \overline{p(\gamma)} d v(\gamma)\right|: p \in \mathfrak{B}_{I}^{2},\|p\| \leq 1\right\} \\
& =\sup \left\{|(g \mid p)|: p \in \mathfrak{B}_{I}^{2},\|p\| \leq 1\right\}=\|g\|<\infty,
\end{aligned}
$$

which is a contradiction.

Q.E.D. 


\section{§3. Construction of the Operator Valued Weight $T_{\alpha}$}

This section is concerned with construction of a faithful normal semifinite operator valued weight of the crossed product by a groupoid action to the algebra on which the groupoid is acting.

Throughout this section, we fix an action $\left(\mathscr{G},\{\mathcal{M}(x)\}_{x \in X},\left\{\alpha_{\gamma}\right\}_{\gamma \in \mathscr{G}}\right\}$ of $\mathscr{G}$. Let $\mathscr{H}(x)$ be the canonical $L^{2}$-space of $M(x)$ in the sense of Kosaki [K]. We set

$$
M=\int_{X}^{\oplus} M(x) d \mu(x), \quad \mathscr{H}=\int_{X}^{\oplus} \mathscr{H}(x) d \mu(x) .
$$

We denote by $u(\gamma)$ the canonical unitary from $\mathscr{H}(s(\gamma))$ onto $\mathscr{H}(r(\gamma))$ implementing the $*$-isomorphism $\alpha_{\gamma}$, that is, $\alpha_{\gamma}=\operatorname{Ad} u(\gamma)$ (see [H1]). From this action, we construct the crossed product algebra $M \times_{\alpha} \mathscr{G}$. We refer readers to [Y1], [Y2] for its construction and the details. On $\mathcal{M} \times{ }_{\alpha} \mathscr{G}$, we have a coaction $\widehat{\alpha}$ of $\mathscr{G}$, called the dual coaction. Since we will deal with the dual coaction intensively in this section, we first recall how we constructed it in [Y1]. The crossed product $\mu \times_{\alpha} \mathscr{G}$ acts on the relative tensor product $\widehat{\mathscr{H}}=\mathscr{H} \otimes_{\mu \mathscr{x}} L^{2}(\mathscr{G}, v)$, which is the direct integral $\int_{X}^{\otimes} \mathscr{H}(x) \otimes L^{2}\left(\mathscr{G}^{x}, \lambda^{x}\right) d \mu(x)$. Note that we can identify $\mathscr{H} \otimes_{\mu \mathscr{x}} L^{2}(\mathscr{G}, v)$ with the set of all functions $\eta$ from $\mathscr{G}$ into $\Pi_{x \in X} \mathscr{H}(x)$ such that (i) $\eta(\gamma) \in \mathscr{H}(r(\gamma)),(\gamma \in \mathscr{G})$; (ii) the function $x \in X \mapsto \int f_{m, x}(\gamma)\left(\xi_{n, x} \mid \eta(\gamma)\right) d \lambda^{x}(\gamma)$ is measurable for any $m, n \in \mathbf{N}$, where $\left\{\xi_{n}\right\}_{n \geq 1}$ and $\left\{f_{m}\right\}_{m \geq 1}$ are fundamental sequences of measurable fields for $\{\mathscr{H}(x)\}$ and $\left\{L^{2}\left(\mathscr{G}^{x}, \lambda^{x}\right)\right\}$, respectively; (iii) $\int\|\eta(\gamma)\|^{2} d v(\gamma)<\infty$. The norm of such a function $\eta$ is defined by $\|\eta\|=\left(\int\|\eta(\gamma)\|^{2} d v(\gamma)\right)^{1 / 2}$. Next we consider the Hilbert space $\widetilde{\mathscr{H}}=\mathscr{H} \otimes_{\mu}$ ${ }_{\mathscr{L}} L^{2}(\mathscr{G}, v) \bigotimes_{\mu \mathscr{L}} L^{2}(\mathscr{G}, v)$. In terms of a direct integral decomposition, we have

$$
\widetilde{\mathscr{H}}=\int_{X}^{\oplus} \mathscr{H}(x) \otimes L^{2}\left(\varphi^{x}, \lambda^{x}\right) \otimes L^{2}\left(\mathscr{G}^{x}, \lambda^{x}\right) d \mu(x) .
$$

Note that we may identify the Hilbert space $\widetilde{\mathscr{H}}$ with the set of all functions $\eta$ from $\mathscr{H}^{(2)}$ into $\Pi_{x \in X} \mathscr{H}(x)$ such that (i) $\eta\left(\gamma_{1}, \gamma_{2}\right) \in \mathscr{H}\left(r\left(\gamma_{1}\right)\right)$ for any $\left(\gamma_{1}, \gamma_{2}\right)$ in $\mathcal{H}^{(2)}$; (ii) the function given by

$$
x \in X \mapsto \iint f_{k, x}\left(\gamma_{1}\right) f_{l, x}\left(\gamma_{2}\right)\left(\xi_{m, x} \mid \eta\left(\gamma_{1}, \gamma_{2}\right)\right) d \lambda^{r\left(\gamma_{1}\right)}\left(\gamma_{2}\right) d \lambda^{x}\left(\gamma_{1}\right)
$$

is measurable for $k, l$ and $m \in \mathbf{N}$, where $\left\{f_{n}\right\}_{n \geq 1}$ and $\left\{\xi_{m}\right\}_{m \geq 1}$ are as before; (iii) $\int\left\|\eta\left(\gamma_{1}, \gamma_{2}\right)\right\|^{2} d v_{1}\left(\gamma_{1}, \gamma_{2}\right)<\infty$. Here $v_{1}$ is the Borel measure on the standard Borel space

$$
\mathscr{H}^{(2)}=\left\{\left(\gamma_{1}, \gamma_{2}\right) \in \mathscr{G} \times \mathscr{G}: r\left(\gamma_{1}\right)=r\left(\gamma_{2}\right)\right\}
$$

determined by the integral

$$
\begin{aligned}
\int_{\mathscr{H}(2)} f\left(\gamma_{1}, \gamma_{2}\right) d v_{1}\left(\gamma_{1}, \gamma_{2}\right) & =\iiint f\left(\gamma_{1}, \gamma_{2}\right) d \lambda^{x}\left(\gamma_{2}\right) d \lambda^{x}\left(\gamma_{1}\right) d \mu(x) \\
& =\iiint f\left(\gamma_{1}, \gamma_{2}\right) d \lambda^{x}\left(\gamma_{1}\right) d \lambda^{x}\left(\gamma_{2}\right) d \mu(x),
\end{aligned}
$$


where $f$ is a positive Borel function on $\mathscr{H}^{(2)}$. The norm of a vector $\eta$ is given by $\|\eta\|=\left(\int\left\|\eta\left(\gamma_{1}, \gamma_{2}\right)\right\|^{2} d v_{1}\left(\gamma_{1}, \gamma_{2}\right)\right)^{1 / 2}$. Similarly, we may identify the Hilbert space $\widetilde{H}_{1}=\mathscr{H} \otimes_{\mu} L^{2}(\mathscr{G}, v)_{\mathscr{L}} \otimes_{\mu \mathscr{L}} L^{2}(\mathscr{G}, v)$ with the set of functions $\zeta$ from $\mathscr{G}^{(2)}$ into $\Pi_{x \in X} \mathscr{H}(x)$ such that (i) $\zeta\left(\gamma_{1}, \gamma_{2}\right) \in \mathscr{H}\left(s\left(\gamma_{1}\right)\right),\left(\left(\gamma_{1}, \gamma_{2}\right) \in \mathscr{G}^{(2)}\right)$; (ii) the function: $\quad x \in X \mapsto \iint \overline{J f_{k, x}}\left(\gamma_{1}\right) f_{l, x}\left(\gamma_{2}\right)\left(\xi_{m, x} \mid \zeta\left(\gamma_{1}, \gamma_{2}\right)\right) d \lambda_{x}^{\prime}\left(\gamma_{1}\right) d \lambda^{x}\left(\gamma_{2}\right) \quad$ is measurable for any $k, l$ and $m \in \mathbf{N}$, where $\left\{f_{n}\right\}_{n \geq 1}$ and $\left\{\xi_{m}\right\}_{m \geq 1}$ are as above, and $J$ is the modular conjugation of $\mathfrak{B}_{I}$; (iii) $\zeta$ is $v_{2}$-square-integrable: $\int\left\|\zeta\left(\gamma_{1}, \gamma_{2}\right)\right\|^{2} d v_{2}\left(\gamma_{1}, \gamma_{2}\right)<\infty$. The measure $v_{2}$ is the Borel measure on the standard Borel space $\mathscr{G}^{(2)}$ defined by the integral

$$
\int_{g(2)} f\left(\gamma_{1}, \gamma_{2}\right) d v_{2}\left(\gamma_{1}, \gamma_{2}\right)=\iiint f\left(\gamma_{1}, \gamma_{2}\right) d \lambda^{s\left(\gamma_{1}\right)}\left(\gamma_{2}\right) d \lambda^{x}\left(\gamma_{1}\right) d \mu(x) \text {. }
$$

The norm of such a function $\zeta$ is $\|\zeta\|=\left(\int\left\|\zeta\left(\gamma_{1}, \gamma_{2}\right)\right\|^{2} d v_{2}\left(\gamma_{1}, \gamma_{2}\right)\right)^{1 / 2}$. It is shown in Lemma 4.3 of [Y1] that there exists a unitary $W_{\mathscr{H}}$ from $\widetilde{\mathscr{H}}_{\text {onto }} \widetilde{\mathscr{H}}_{1}$ defined by

$$
\left\{W_{\mathscr{H}} \eta\right\}\left(\gamma_{1}, \gamma_{2}\right)=u\left(\gamma_{1}\right)^{+} \eta\left(\gamma_{1}, \gamma_{1} \gamma_{2}\right) \quad\left(\eta \in \widetilde{\mathscr{H}},\left(\gamma_{1}, \gamma_{2}\right) \in \mathscr{G}^{(2)}\right) .
$$

The inverse is given by

$$
\left\{W_{\mathscr{H}}^{+} \zeta\right\}\left(\gamma_{1}, \gamma_{2}\right)=u\left(\gamma_{1}\right) \zeta\left(\gamma_{1}, \gamma_{1}^{-1} \gamma_{2}\right) \quad\left(\zeta \in \widetilde{\mathscr{H}}_{1},\left(\gamma_{1}, \gamma_{2}\right) \in \mathscr{H}^{(2)}\right) .
$$

We need to introduce one more unitary to define the dual coaction. Note that there is a $\mathscr{L}$ action on $\widehat{\mathscr{H}}$ given by $h \in \mathscr{L} \mapsto 1 \bigotimes_{\mathscr{L}} M(h \circ s)$. Let us write $\widehat{\mathscr{H}}_{\mathscr{L}}$ for $\widehat{\mathscr{H}}$ if this action is particularly considered. The above action commutes with that of $M \times_{\alpha} \mathscr{G}$. So we may form the relative tensor product of $\widehat{\mathscr{H}}_{\mathscr{L}}$ and ${ }_{\mathscr{L}} L^{2}(\mathscr{G}, v)$. Suppose that $\xi \in \widehat{\mathscr{H}}$ and $f \in D\left({ }_{\mathscr{L}} L^{2}(\mathscr{G}, v), \mu\right)$. We denote by $\xi \widehat{\otimes}_{\mu} f$ the elementary tensor of $\xi$ and $f$ in $\widehat{\mathscr{H}}_{\mathscr{Q}} \otimes_{\mu \mathscr{L}} L^{2}(\mathscr{G}, v)$. Then it is observed in [Y1] that the equation

$$
\left\{V_{\mathscr{H}}\left(\xi \widehat{\otimes}_{\mu} f\right)\right\}\left(\gamma_{1}, \gamma_{2}\right)=f\left(\gamma_{2}\right) u\left(\gamma_{1}\right)^{+} \xi\left(\gamma_{1}\right)
$$

defines a unitary from $\widehat{\mathscr{H}}_{\mathscr{L}} \otimes_{\mu \mathscr{L}} L^{2}(\mathscr{G}, v)$ onto $\widetilde{\mathscr{H}}_{1}$. Now, since the $\mathscr{E}$ action on $\widehat{\mathscr{H}}_{\mathscr{L}}$ commutes with that of $M \times_{\alpha} G$, it makes a perfect sense to form $y \otimes_{\mathscr{L}} 1$ on $\widehat{\mathscr{H}}_{P_{X}} \otimes_{\mu} L^{2}(\mathscr{G}, v)$ for $y \in M \times_{\alpha} \mathscr{G}$. (We hereafter write $y \widehat{\otimes}_{\mathscr{Z}} 1$ for the above tensor product so as to distinguish it from the relative tensor products on $\left.\widetilde{\mathscr{H}}=\widehat{\mathscr{H}} \otimes_{\mu \mathscr{L}} L^{2}(\mathscr{G}, v)\right)$. We define a $*$-isomorphism $\widehat{\alpha}$ from $\mathcal{M} \times_{\alpha} \mathscr{G}$ into $\mathscr{L}(\widetilde{\mathscr{H}})=$ the set of all bounded operators on $\mathscr{H}$ by

$$
\widehat{\alpha}(y)=W_{\mathscr{H}}^{+} V_{\mathscr{H}}\left(y \widehat{\otimes}_{\mathscr{L}} 1\right) V_{\mathscr{H}}^{+} W_{\mathscr{H}} \quad\left(y \in M \times_{\alpha} \varphi\right) .
$$

This morphism $\widehat{\alpha}$ serves as the dual coaction of $\mathscr{G}$ on the crossed product $M \times{ }_{\alpha} \mathscr{G}$.

Set $\mathscr{L}_{\mathscr{L}}(\widehat{\mathscr{H}})=\mathscr{L}(\widehat{\mathscr{H}}) \cap\left\{1 \bigotimes_{\mathscr{L}} M(h \circ s): h \in \mathscr{E}\right\}^{\prime}$. For any $Y \in \mathscr{L}_{\mathscr{L}_{s}}(\widehat{\mathscr{H}})$, we put

$$
\Pi(Y)=W_{\mathscr{H}}^{+} V_{\mathscr{H}}\left(Y \widehat{\otimes}_{\mathscr{L}} 1\right) V_{\mathscr{H}}^{+} W_{\mathscr{H}},
$$

where the operator $Y \widehat{\otimes}_{\mathscr{L}} 1$, of course, denotes the relative tensor product of $Y$ and 1 on $\widehat{\mathscr{H}}_{\mathscr{L}} \otimes_{\mu \mathscr{L}} L^{2}(\mathscr{G}, v)$. Then $\Pi$ is a $*$-isomorphism from $\widehat{\mathscr{L}}_{\mathscr{L}}(\mathscr{H})$ into $\widehat{\mathscr{L}}(\mathscr{H})$. 
We note that the restriction of $\Pi$ to the crossed product $M \times_{\alpha} \varphi$ is nothing but the dual coaction $\widehat{\alpha}$ of $\mathscr{G}$. We now consider the von Neumann subalgebra $\mathscr{L}(\mathscr{H}) *_{\mathscr{L}} L^{\infty}(\mathscr{G}, v)=\mathscr{L}_{\mathscr{L}}(\mathscr{H}) \bigotimes_{\mathscr{L}} L^{\infty}(\mathscr{G}, v)$ of $\mathscr{L}_{\mathscr{L}_{s}}(\widehat{\mathscr{H}})$, where $\mathscr{L}_{\mathscr{L}}(\mathscr{H})=\mathscr{L}(\mathscr{H}) \cap \mathscr{L}^{\prime}=$ the set of all decomposable operators on $\mathscr{H}=\int_{X}^{\oplus} \mathscr{H}(x) d \mu(x)$.

Lemma 3.1. With the above notations, we have

$$
\Pi\left(Y_{0}\right)=Y_{0} \otimes_{\mathscr{L}} 1
$$

for any $Y_{0} \in \mathscr{L}(\mathscr{H}) *_{\mathscr{L}} L^{\infty}(\mathscr{G}, v)$.

Proof. Let $T=\int_{X}^{\oplus} T(x) d \mu(x) \in \mathscr{L}_{\mathscr{L}}(\mathscr{H})$ and $k \in L^{\infty}(\mathscr{G}, v)$. Then, for any $\xi \in \mathscr{H}, f \in D\left(\mathscr{L} L^{2}(\mathscr{G}, v), \mu\right)$ and any $\left(\gamma_{1}, \gamma_{2}\right) \in \mathscr{H}^{(2)}$, we have

$$
\begin{aligned}
& \left\{\left(T \otimes_{\mathscr{L}} M(k) \bigotimes_{\mathscr{L}} 1\right) W_{\mathscr{H}}^{+} V_{\mathscr{H}}\left(\xi \widehat{\otimes}_{\mu} f\right)\right\}\left(\gamma_{1}, \gamma_{2}\right) \\
& =k\left(\gamma_{1}\right) T\left(r\left(\gamma_{1}\right)\right)\left\{W_{\mathscr{H}}^{i} V_{\mathscr{H}}\left(\xi \widehat{\otimes}_{\mu} f\right)\right\}\left(\gamma_{1}, \gamma_{2}\right) \\
& =k\left(\gamma_{1}\right) T\left(r\left(\gamma_{1}\right)\right) u\left(\gamma_{1}\right)\left\{V_{\mathscr{H}}\left(\xi \widehat{\otimes}_{\mu} f\right)\right\}\left(\gamma_{1}, \gamma_{1}^{-1} \gamma_{2}\right) \\
& =k\left(\gamma_{1}\right) f\left(\gamma_{1}^{-1} \gamma_{2}\right) T\left(r\left(\gamma_{1}\right)\right) \xi\left(\gamma_{1}\right) .
\end{aligned}
$$

We also have

$$
\begin{aligned}
& \left\{W_{\mathscr{H}}^{+} V_{\mathscr{H}}\left(\left(T \bigotimes_{\mathscr{L}} M(k)\right) \widehat{\otimes}_{\mathscr{L}} 1\right)\left(\xi \widehat{\otimes}_{\mu} f\right)\right\}\left(\gamma_{1}, \gamma_{2}\right) \\
& =u\left(\gamma_{1}\right)\left\{V_{\mathscr{H}}\left(\left(T \bigotimes_{\mathscr{L}} M(k)\right) \xi \widehat{\bigotimes}_{\mu} f\right)\right\}\left(\gamma_{1}, \gamma_{1}^{-1} \gamma_{2}\right) \\
& =u\left(\gamma_{1}\right) f\left(\gamma_{1}^{-1} \gamma_{2}\right) u\left(\gamma_{1}\right)^{+}\left\{\left(T \bigotimes_{\mathscr{L}} M(k)\right) \xi\right\}\left(\gamma_{1}\right) \\
& =k\left(\gamma_{1}\right) f\left(\gamma_{1}^{-1}\right) T\left(r\left(\gamma_{1}\right)\right) \xi\left(\gamma_{1}\right) .
\end{aligned}
$$

These calculations show that $\Pi\left(T \bigotimes_{\mathscr{L}} M(k)\right)=T \bigotimes_{\mathscr{L}} M(k) \bigotimes_{\mathscr{L}} 1$. Thus we obtain the desired identity.

Q.E.D.

In what follows, we let $\mathscr{E}^{+}(\mathscr{P})$ denote the extended positive part of a von Neumann algbera $\mathscr{P}$ in the sense of Haagerup [H2].

For each $Y \in \mathscr{L}_{\mathscr{L}_{s}}(\widehat{\mathscr{H}})_{+}$, we define a map $S(Y)$ on $\mathscr{L}(\widehat{\mathscr{H}})_{+}^{+}$by

$$
\begin{aligned}
S(Y)(\omega) & =\left\langle\Pi(Y), \omega *_{\mu} \varphi_{g}\right\rangle \quad\left(\omega \in \mathscr{L}(\widehat{\mathscr{H}})_{+}^{+}\right) \\
& =\sup _{h \in \mathscr{P}(\mathscr{G})}\left\langle\Pi(Y), \omega *_{\mu} \omega_{h}\right\rangle .
\end{aligned}
$$

Then it is easy to see that $S(Y)$ in an element of $\mathscr{E}^{+}(\mathscr{L}(\widehat{\mathscr{H}}))$. We note that $S$ is additive, homogeneous and normal. Moreover, due to Lemma 3.1, we have

$$
S\left(Y_{0}^{+} Y Y_{0}\right)=Y_{0}^{+} S(Y) Y_{0}
$$

for any $Y_{0} \in \mathscr{L}(\mathscr{H}) *_{\mathscr{L}} L^{\infty}(\mathscr{G}, v)$ and $Y \in \mathscr{L}_{\mathscr{E}_{s}}(\widehat{\mathscr{H}})$. Indeed, 


$$
\begin{aligned}
S\left(Y_{0}^{+} Y Y_{0}\right)(\omega) & =\sup _{h \in \mathscr{P}(\mathscr{g})}\left\langle\Pi\left(Y_{0}^{+} Y Y_{0}\right), \omega *_{\mu} \omega_{h}\right\rangle \\
& =\sup _{h \in \mathscr{P}(\mathscr{g})}\left\langle\left(Y_{0}^{+} \bigotimes_{\mathscr{L}} 1\right) \Pi(Y)\left(Y_{0} \otimes_{\mathscr{I}} \mathbb{1}\right), \omega *_{\mu} \omega_{h}\right\rangle \\
& =\sup _{h \in \mathscr{P}(\mathscr{g})}\left\langle\Pi(Y), Y_{0} \omega Y_{0}^{+} *_{\mu} \omega_{h}\right\rangle \\
& =S(Y)\left(Y_{0} \omega Y_{0}^{+}\right)=Y_{0}^{*} S(Y) Y_{0}(\omega) .
\end{aligned}
$$

Let $P$ be a unitary from $\mathscr{H} \otimes_{\mu} L^{2}(\mathscr{G}, v)_{\mathscr{L}}$ onto $\mathscr{H}$ defined by

$$
\{P \eta\}(\gamma)=u(\gamma) \eta(\gamma) \quad\left(\eta \in \mathscr{H} \otimes_{\mu} L^{2}(G, v)_{\mathscr{L}}\right) .
$$

Here $\mathscr{H} \otimes_{\mu} L^{2}(\mathscr{G}, v)_{\mathscr{L}}$ is regarded as a set of functions $\eta$ from $\mathscr{G}$ into $\Pi_{x \in X} \mathscr{H}(x)$ satisfying $\eta(\gamma) \in \mathscr{H}(s(\gamma))$ and some other conditions (see [Y1]). We note that $P$ is a $\mathscr{E}$-module map from $\mathscr{H}_{\mu} \otimes^{2}(\mathscr{G}, v)_{\mathscr{E}}$ onto $\widehat{\mathscr{H}}_{\mathscr{X}}$. Hence $P^{+} \mathscr{L}_{\mathscr{E}}(\widehat{\mathscr{H}}) P$ is the set of all decomposable operators on $\mathscr{H} \otimes_{\mu} L^{2}(\mathscr{G}, v)_{\mathscr{L}}$. Let $\eta_{i}=\int_{X}^{\oplus} \eta_{i, x} d \mu(x)(i=1,2)$ be $\mu$-bounded vectors in $\mathscr{H} \otimes_{\mu} L^{2}(\mathscr{G}, v)_{\mathscr{X}}=\int_{X}^{\oplus} \mathscr{H}(x) \otimes L^{2}\left(\mathscr{G}_{x}, \lambda_{x}^{\prime}\right) d \mu(x)$. For each $x \in X$, we denote by $t_{\eta_{1}, \eta_{2}}(x)$ the operator of rank one given by

$$
t_{\eta_{1}, \eta_{2}}(x)(\zeta)=\left(\zeta \mid \eta_{2, x}\right) \eta_{1, x} \quad\left(\zeta \in \mathscr{H}(x) \otimes L^{2}\left(\mathscr{G}_{x}, \lambda_{x}^{\prime}\right)\right)
$$

It is easy to see that the family $\left\{t_{\eta_{1}, \eta_{2}}(x)\right\}_{x \in X}$ is a bounded measurable field of operators. Thus it defines a decomposable operator $t_{\eta_{1}, \eta_{2}}$ on $\mathscr{H} \otimes_{\mu} L^{2}(\mathscr{G}, v)_{\mathscr{T}}$ by

$$
t_{\eta_{1}, \eta_{2}}=\int_{X}^{\oplus} t_{\eta_{1}, \eta_{2}}(x) d \mu(x)
$$

We write $t_{\eta}$ for $t_{\eta, \eta}$. Set

$$
\Phi=\operatorname{span}\left\{t_{\eta_{1}, \eta_{2}}: \eta_{i} \text { 's are as above. }\right\} .
$$

Then $\Phi$ is a $\sigma$-weakly dense two-sided ideal in the set of decomposable operators. Consequently, $P \mathscr{F} P^{+}$is a $\sigma$-weakly dense two-sided ideal of $\mathscr{L}_{\mathscr{Q}}(\widehat{\mathscr{H}})$. If $\eta$ is a vector in $D\left(\mathscr{H} \otimes_{\mu} L^{2}(\mathscr{G}, v)_{\mathscr{L}}, \mu\right)$, then, for any $\zeta \in \mathscr{H} \otimes_{\mu} L^{2}(\mathscr{G}, v)_{\mathscr{L}}$, we have

$$
\left\{t_{\eta} \zeta\right\}(\gamma)=\int\left(\zeta\left(\gamma_{1}\right) \mid \eta\left(\gamma_{1}\right)\right) d \lambda_{s(\gamma)}^{\prime}\left(\gamma_{1}\right) \eta(\gamma) .
$$

Thus the operator $P t_{\eta} P^{+}$acts on $\widehat{\mathscr{H}}$ in the following manner:

$$
\left\{P t_{\eta} P^{+} \xi\right\}(\gamma)=\int\left(\xi\left(\gamma_{1}\right) \mid\{P \eta\}\left(\gamma_{1}\right)\right) d \lambda_{s(\gamma)}^{\prime}\left(\gamma_{1}\right)\{P \eta\}(\gamma)
$$

for any $\xi \in \widehat{\mathscr{H}}$.

Lemma 3.3. Let $\eta$ be as above. Then, for any $\zeta \in \widetilde{\mathscr{H}}$, we have

$$
\left\{\Pi\left(P t_{\eta} P^{+}\right) \zeta\right\}\left(\gamma_{1}, \gamma_{2}\right)=\int\left(\zeta\left(\gamma, \gamma \gamma_{1}^{-1} \gamma_{2}\right) \mid\{P \eta\}(\gamma)\right) d \lambda_{s\left(\gamma_{1}\right)}^{\prime}(\gamma)\{P \eta\}\left(\gamma_{1}\right) \text {. }
$$


Proof. Let $A_{\eta}$ denote the operator defined by the right side of the above assertion. For any $\xi \in \widehat{\mathscr{H}}, f \in D\left(\mathscr{x} L^{2}(\mathscr{G}, v), \mu\right)$ and $\left(\gamma_{1}, \gamma_{2}\right) \in \mathscr{H}^{(2)}$, we compute

$$
\begin{aligned}
& \left\{W_{\mathscr{H}}^{+} V_{\mathscr{H}}\left(P t_{\eta} P^{+} \widehat{\otimes}_{\mathscr{L}} 1\right)\left(\xi \widehat{\otimes}_{\mu} f\right)\right\}\left(\gamma_{1}, \gamma_{2}\right) \\
& =u\left(\gamma_{1}\right)\left\{V_{\mathscr{H}}\left(P t_{\eta} P^{+} \xi \widehat{\otimes}_{\mu} f\right)\right\}\left(\gamma_{1}, \gamma_{1}^{-1} \gamma_{2}\right) \\
& =f\left(\gamma_{1}^{-1} \gamma_{2}\right)\left\{P t_{\eta} P^{+} \xi\right\}\left(\gamma_{1}\right) \\
& =f\left(\gamma_{1}^{-1} \gamma_{2}\right) \int(\xi(\gamma) \mid\{P \eta\}(\gamma)) d \lambda_{s\left(\gamma_{1}\right)}^{\prime}(\gamma)\{P \eta\}\left(\gamma_{1}\right) .
\end{aligned}
$$

We also have

$$
\begin{aligned}
& \left\{A_{\eta} W_{\mathscr{H}}^{+} V_{\mathscr{H}}\left(\xi \widehat{\otimes}_{\mu} f\right)\right\}\left(\gamma_{1}, \gamma_{2}\right) \\
& =\int\left(\left\{W_{\mathscr{H}}^{+} V_{\mathscr{H}}\left(\xi \widehat{\otimes}_{\mu} f\right)\right\}\left(\gamma, \gamma \gamma_{1}^{-1} \gamma_{2}\right) \mid\{P \eta\}(\gamma)\right) d \lambda_{s\left(\gamma_{1}\right)}^{\prime}(\gamma)\{P \eta\}\left(\gamma_{1}\right) \\
& =\int\left(u(\gamma)\left\{V_{\mathscr{H}}\left(\xi \widehat{\otimes}_{\mu} f\right)\right\}\left(\gamma, \gamma_{1}^{-1} \gamma_{2}\right) \mid\{P \eta\}(\gamma)\right) d \lambda_{s\left(\gamma_{1}\right)}^{\prime}(\gamma)\{P \eta\}\left(\gamma_{1}\right) \\
& =\int\left(f\left(\gamma_{1}^{-1} \gamma_{2}\right) \xi(\gamma) \mid\{P \eta\}(\gamma)\right) d \lambda_{s\left(\gamma_{1}\right)}^{\prime}(\gamma)\{P \eta\}\left(\gamma_{1}\right) .
\end{aligned}
$$

It follows that $W_{\mathscr{H}}^{+} V_{\mathscr{H}}\left(P t_{\eta} P^{+} \widehat{\bigotimes}_{\mathscr{L}} 1\right)=A_{\eta} W_{\mathscr{H}}^{+} V_{\mathscr{H}}$. Therefore $\Pi\left(P t_{\eta} P^{+}\right)=A_{\eta}$.

Proposition 3.4. The map $S$ is a normal operator valued weight from $\mathscr{L}_{\mathscr{E}_{5}}(\widehat{\mathscr{H}})$ onto $\mathscr{L}(\mathscr{H}) *_{\mathscr{L}} L^{x}(\mathscr{G}, v)$.

Proof. Let $\eta \in D\left(\mathscr{H} \otimes_{\mu} L^{2}(\mathscr{G}, v)_{\mathscr{L}}, \mu\right), \xi \in \widehat{\mathscr{H}}$ and $h \in \mathscr{P}(\mathscr{G})$. Then, by Lemma 3.3 and Fubini's theorem, we get

$$
\begin{aligned}
& \left\langle\Pi\left(P t_{\eta} P^{+}\right), \omega_{\xi} *_{\mu} \omega_{h}\right\rangle \\
& =\left\langle\Pi\left(P t_{\eta} P^{+}\right), \omega_{\xi \otimes_{u} h}\right\rangle \\
& =\left(\Pi\left(P t_{\eta} P^{+}\right)\left(\xi \otimes_{\mu} h\right) \mid \xi \otimes_{\mu} h\right) \\
& =\int\left(\left\{\Pi\left(P t_{\eta} P^{+}\right)\left(\xi \otimes_{\mu} h\right)\right\}\left(\gamma_{1}, \gamma_{2}\right) \mid\left(\xi \otimes_{\mu} h\right)\left(\gamma_{1}, \gamma_{2}\right)\right) d v_{1}\left(\gamma_{1}, \gamma_{2}\right) \\
& =\int\left(\int h\left(\gamma \gamma_{1}^{-1} \gamma_{2}\right)(\xi(\gamma) \mid\{P \eta\}(\gamma)) d \lambda_{s\left(\gamma_{1}\right)}^{\prime}(\gamma)\right)\left(\{P \eta\}\left(\gamma_{1}\right) \mid \xi\left(\gamma_{1}\right)\right) \overline{h\left(\gamma_{2}\right)} d v_{1}\left(\gamma_{1}, \gamma_{2}\right) \\
& =\int\left(q * q^{\#}\right)(\gamma) \overline{\left(h * h^{b}\right)(\gamma)} d v(\gamma),
\end{aligned}
$$

where $q(\gamma)=(\{P \eta\}(\gamma) \mid \xi(\gamma))(\gamma \in G)$. By Proposition 2.8, we have

$$
S\left(P t_{\eta} P^{+}\right)\left(\omega_{\xi}\right)=\int\left|q^{\#}(\gamma)\right|^{2} d v(\gamma)=\int \delta(\gamma)|(\{P \eta\}(\gamma) \mid \xi(\gamma))|^{2} d v(\gamma)
$$

It follows from (3.2) that, for any $k \in L^{\infty}(\mathscr{G}, v)$ with $|k|=1$, we have 


$$
\begin{aligned}
& \left(1 \otimes_{\mathscr{L}} M(k)^{+}\right) S\left(P t_{\eta} P^{+}\right)\left(1 \bigotimes_{\mathscr{L}} M(k)\right)\left(\omega_{\xi}\right) \\
& =S\left(P t_{\eta} P^{+}\right)\left(\omega_{\left.\left(1 \otimes_{\xi} M(k)\right) \xi\right)}\right. \\
& =\int \delta(\gamma) \mid\left(\{P \eta\}(\gamma) \mid\left\{\left(1 \bigotimes_{\mathscr{L}} M(k)\right) \xi\right\}(\gamma)\right) d v(\gamma) \\
& =\int \delta(\gamma)|(\{P \eta\}(\gamma) \mid k(\gamma) \xi(\gamma))|^{2} d v(\gamma) \\
& =\int \delta(\gamma)|(\{P \eta\}(\gamma) \mid \xi(\gamma))|^{2} d v(\gamma) \\
& =S\left(P t_{\eta} P^{+}\right)\left(\omega_{\xi}\right) .
\end{aligned}
$$

Hence $S\left(P t_{\eta} P^{+}\right)$is affiliated with $\left\{\mathbf{C} \otimes_{\mathscr{L}} L^{\infty}(\mathscr{G}, v)\right\}^{\prime}=\mathscr{L}(\mathscr{H}) *_{\mathscr{L}} L^{\infty}(\mathscr{G}, v)$ (c.f. Definition 1.8 of [H2]). Since the set of operators $P t_{n} P^{+}$is the positive part of the $\sigma$-weakly dense two-sided ideal $P \mathscr{I} P^{+}$in $\mathscr{L}_{\mathscr{L}_{5}}(\widehat{\mathscr{H}})$, every $Y \in \mathscr{L}_{\mathscr{L}}(\widehat{\mathscr{H}})_{+}$can be written as the limit of an increasing net of elements of the form $P t_{\eta} P^{+}$. It follows from the normality of $S$ that $S(Y)$ is affiliated with $\mathscr{L}(\mathscr{H}) *{ }_{\mathscr{L}} L^{\infty}(\mathscr{G}, v)$ for every $Y \in \mathscr{L}_{\mathscr{L}_{S}}(\widehat{\mathscr{H}})_{+}$. So the map $S$ is a normal operator valued weight from $\mathscr{L}_{\mathscr{L}_{3}}(\widehat{\mathscr{H}})$ onto $\mathscr{L}(\mathscr{H}) *_{\text {o }} L^{\nsim}(\mathscr{G}, v)$.

Q.E.D.

In order to obtain a faithful normal semifinite operator valued weight from $M \times_{\alpha} G$ to $M \otimes_{\mathscr{P}} \mathbb{C}$, we just restrict the map $S$ to the crossed product. It is not so difficult to show that the restriction is faithful and normal, except that it is semifinite. For semifiniteness, we need to prepare a few lemmas.

Let $f \in \mathfrak{B}_{I}$ and $\xi \in \widehat{\mathscr{H}}$. Then we consider that vector $f \otimes_{\mu} \xi$ in $L^{2}(\mathscr{G}, v)_{\mathcal{L}_{\mathcal{L}}} \otimes_{\mu} \widehat{\mathscr{H}}$. Since the operation of taking relative tensor products of Hilbert spaces is associative and commutative, the space $L^{2}(\mathscr{G}, v)_{\mathscr{X}} \otimes_{\mu} \widehat{\mathscr{H}}$ can be identified with $\widehat{\mathscr{H}}_{1}$. (In other words, there exists a $\mathscr{L}$-module unitary from $L^{2}(\mathscr{G}, v)_{\mathscr{L}} \otimes_{\mu} \widehat{\mathscr{H}}$ onto $\left.\widehat{\mathscr{H}}_{1}\right)$. We denote by $f \times_{\mu} \xi$ the vector $f \otimes_{\mu} \xi$ in $\widehat{\mathscr{H}}_{1}$ under this identification.

In what follows, we will consider the operator $(u \otimes \lambda)(g)\left(g \in \mathfrak{B}_{I}\right)$ on $\widehat{\mathscr{H}}$, which is a typical element in the crossed product. We refer readers to Lemma 2.10 of [Y2] for the notation $(u \otimes \lambda)(\mathrm{g})$. It is shown in Lemma 4.4 of [Y1] that $\widehat{\alpha}((u \otimes \lambda)(g))=(u \otimes \lambda \otimes \lambda)(g)$, where $(u \otimes \lambda \otimes \lambda)(\cdot)$ is the integrated form of the representation $u(\gamma) \otimes \lambda(\gamma) \otimes \lambda(\gamma)$ of $\varphi$ on the Hilbert bundle $\{\mathscr{H}(x) \otimes$ $\left.L^{2}\left(\mathscr{G}^{x}, \lambda^{x}\right) \otimes L^{2}\left(\mathscr{G}^{x}, \lambda^{x}\right)\right\}_{x \in X}$.

Lemma 3.5. Let $f \in \mathfrak{B}_{I}^{\infty}$ and $\xi \in \widehat{\mathscr{H}}$. Then

$$
\left\|\widehat{\alpha}((u \otimes \lambda)(f))\left(\xi \otimes_{\mu} h\right)\right\| \leq\left\|\pi_{r}(h)\right\|\left\|f \times_{\mu} \xi\right\|
$$

for any right bounded vector $h \in D\left({ }_{2} L^{2}(\varphi, v), \mu\right)$.

Proof. Let $k \in L^{2}(\mathscr{G}, v)$ and $\eta$ be an element in $\widehat{\mathscr{H}}$ such that the function $f_{\eta}$ defined by $f_{\eta}(\gamma)=\|\eta(\gamma)\|(\gamma \in \mathscr{G})$ belongs to $\mathfrak{L}_{l}$. Note that the set of such vectors $\eta$ is dense in $\widehat{\mathscr{H}}$. By Fubini's theorem, we get 


$$
\left(\widehat{\alpha}((u \otimes \lambda)(f))\left(\xi \otimes_{\mu} h\right) \mid \eta \otimes_{\mu} k\right)=\int\{(f \cdot p) * h\}(\gamma) \overline{k(\gamma)} d v(\gamma),
$$

where $p(\gamma)=\int\left(u(\gamma) \xi\left(\gamma^{-1} \gamma_{1}\right) \mid \eta\left(\gamma_{1}\right)\right) d \lambda^{r(\gamma)}\left(\gamma_{1}\right)$. We claim that the function $f \cdot p$ belongs to $L^{2}(\mathscr{G}, v)$. Indeed, we have

$$
|p(\gamma)| \leq \int\left\|\xi\left(\gamma^{-1} \gamma_{1}\right)\right\|\left\|\eta\left(\gamma_{1}\right)\right\| d \lambda^{r(\gamma)}\left(\gamma_{1}\right)=\left(f_{\eta} * f_{\xi}^{b}\right)(\gamma),
$$

where $f_{5}(\gamma)=\|\xi(\gamma)\|$. Then, due to Schwarz inequality and Fubini's theorem,

$$
\begin{aligned}
& \int\left|f(\gamma)\left(f_{\eta} * f_{\xi}^{b}\right)(\gamma)\right|^{2} d v(\gamma) \\
& \leq \int|f(\gamma)|^{2}\left(\int f_{\eta}\left(\gamma_{1}\right) d \lambda^{r(\gamma)}\left(\gamma_{1}\right)\right)\left(\int f_{\eta}\left(\gamma_{1}\right) f_{\xi}\left(\gamma^{-1} \gamma_{1}\right)^{2} d \lambda^{r(\gamma)}\left(\gamma_{1}\right)\right) d v(\gamma) \\
& \leq\left\|f_{\eta}\right\|_{I} \int|f(\gamma)|^{2}\left(\int f_{\eta}\left(\gamma_{1}\right) f_{\xi}\left(\gamma^{-1} \gamma_{1}\right)^{2} d \lambda^{r(\gamma)}\left(\gamma_{1}\right)\right) d v(\gamma) \\
& =\left\|f_{\eta}\right\|_{I} \int|f(\gamma)|^{2}\left(\int f_{\eta}\left(\gamma \gamma_{1}\right) f_{\xi}\left(\gamma_{1}\right)^{2} d \lambda^{s(\gamma)}\left(\gamma_{1}\right)\right) \delta(\gamma) d \lambda_{x}(\gamma) d \mu(x) \\
& =\left\|f_{\eta}\right\|_{I} \int f_{\xi}\left(\gamma_{1}\right)^{2}\left(\int|f(\gamma)|^{2} f_{\eta}\left(\gamma \gamma_{1}\right) \delta(\gamma) d \lambda_{r\left(\gamma_{1}\right)}(\gamma)\right) d v\left(\gamma_{1}\right) .
\end{aligned}
$$

Suppose that $f$ is $\delta_{a}$-bounded. Then

$$
\begin{aligned}
\int|f(\gamma)|^{2} f_{\eta}\left(\gamma \gamma_{1}\right) \delta(\gamma) d \lambda_{r\left(\gamma_{1}\right)}(\gamma) & \leq a\|f\|_{\infty}^{2} \int f_{\eta}\left(\gamma \gamma_{1}\right) d \lambda_{r\left(\gamma_{1}\right)}(\gamma) \\
& =a\|f\|_{\infty}^{2} \int f_{\eta}\left(\gamma^{-1}\right) d \lambda^{s\left(\gamma_{1}\right)}(\gamma) \\
& \leq a\|f\|_{\infty}^{2}\left\|f_{\eta}^{b}\right\|_{I .}
\end{aligned}
$$

Thus we have

$$
\int\left|f(\gamma)\left(f_{\eta} * f_{\xi}^{b}\right)(\gamma)\right|^{2} d v(\gamma) \leq a\|f\|_{\infty}^{2}\left\|f_{\eta}\right\|_{I}\left\|f_{\eta}^{b}\right\|_{I}\|\xi\|^{2}<\infty .
$$

This shows that $f \cdot p \in L^{2}(\varphi, v)$. It follows that (3.6) can be written as

$$
\left(\widehat{\alpha}((u \otimes \lambda)(f))\left(\xi \otimes_{\mu} h\right) \mid \eta \otimes_{\mu} k\right)=\left(\pi_{r}(h) f \cdot p \mid k\right) .
$$

Meanwhile, the vector $W_{\mathscr{H}}^{+}\left(f \times_{\mu} \xi\right)$ falls in $\widetilde{\mathscr{H}}$. If $\sigma$ denotes the unitary from $L^{2}\left(\mathscr{H}^{(2)}, v_{1}\right)$ onto itself induced by the rule $\left(\gamma_{1}, \gamma_{2}\right) \in \mathscr{H}^{(2)} \mapsto\left(\gamma_{2}, \gamma_{1}\right) \in \mathscr{H}^{(2)}$, then $\left(1 \otimes_{\mathscr{X}} \sigma\right) W_{\mathscr{H}}^{+}\left(f \times_{\mu} \xi\right)$ still lies in $\widetilde{\mathscr{H}}$. Fubini's theorem then yields the identity

$$
\left(\left(1 \otimes_{\mathscr{L}} \sigma\right) W_{\mathscr{H}}^{4}\left(f \times_{\mu} \xi\right) \mid \eta \otimes_{\mu} k\right)=(f \cdot p \mid k)
$$

for $\eta, k$ as before. Let us take a sequence $\left\{h_{n}\right\}_{n \geq 1}$ in $\mathfrak{B}_{I}$ with $\left\|\pi_{r}\left(h_{n}\right)\right\| \leq 1$ such that $\left\{\pi_{r}\left(h_{n}\right)\right\}$ converges $\sigma$-strongly to 1 . It follows from (3.7) and (3.8) that 


$$
\begin{aligned}
\lim _{n \rightarrow \infty}\left(\widehat{\alpha}((u \otimes \lambda)(f))\left(\xi \otimes_{\mu} h_{n}\right) \mid \eta \otimes_{\mu} k\right) & =\lim _{n \rightarrow \infty}\left(\pi_{r}\left(h_{n}\right) f \cdot p \mid k\right) \\
& =\left(\left(1 \bigotimes_{\mathscr{T}} \sigma\right) W_{\eta}^{+}\left(f \times_{\mu} \xi\right) \mid \eta \otimes_{\mu} k\right) .
\end{aligned}
$$

Let $g \in \mathfrak{B}_{I}$. In view of (3.7) and (3.9), we have

$$
\begin{aligned}
& \left(\left(1 \otimes_{\mathscr{X}} \pi_{r}(h)\right)\left(1 \otimes_{\mathscr{X}} \sigma\right) W_{\mathscr{H}}^{+}\left(f \times_{\mu} \xi\right) \mid \eta \otimes_{\mu} g\right) \\
& =\left(\left(1 \bigotimes_{\mathscr{X}} \sigma\right) W_{\mathscr{H}}^{+}\left(f \times_{\mu} \xi\right) \mid \eta \otimes_{\mu} \pi_{r}(h)^{+} g\right) \\
& =\lim _{n \rightarrow \infty}\left(\widehat{\alpha}((u \otimes \lambda)(f))\left(\xi \bigotimes_{\mu} h_{n}\right) \mid \eta \bigotimes_{\mu} \pi_{r}(h)^{+} g\right) \\
& =\lim _{n \rightarrow \infty}\left(\widehat{\alpha}((u \otimes \lambda)(f))\left(\xi \bigotimes_{\mu} h_{n}\right) \mid\left(1 \bigotimes_{\mathscr{X}} \pi_{r}(h)^{*}\right)\left(\eta \otimes_{\mu} g\right)\right) \\
& =\lim _{n \rightarrow \infty}\left(\widehat{\alpha}((u \otimes \lambda)(f))\left(\xi \bigotimes_{\mu} h_{n} * h\right) \mid \eta \bigotimes_{\mu} g\right) \\
& =\lim _{n \rightarrow \infty}\left(\pi_{r}\left(h_{n} * h\right) f \cdot p \mid g\right) \\
& =\left(\pi_{r}(h) f \cdot p \mid g\right) \\
& =\left(\widehat{\alpha}((u \otimes \lambda)(f))\left(\xi \bigotimes_{\mu} h\right) \mid \eta \otimes_{\mu} g\right) .
\end{aligned}
$$

The fourth equality is due to commutativity of operators $\widehat{\alpha}((u \otimes \lambda)(f))$ and $1 \otimes_{\mathscr{P}} \pi_{r}(h)$. The computation shows that

$$
\widehat{\alpha}((u \otimes \lambda)(f))\left(\xi \bigotimes_{\mu} h\right)=\left(1 \bigotimes_{\mathscr{X}} \pi_{r}(h)\right)\left(1 \bigotimes_{\mathscr{X}} \sigma\right) W_{\mathscr{H}}^{+}\left(f \times_{\mu} \xi\right) .
$$

The assertion of the lemma is an easy consequence of this identity.

Q.E.D.

Lemma 3.10. Let $f$ and $\xi$ be as in the previous lemma. Then

$$
\sup _{h \in \mathscr{P}(\mathscr{g})}\left\|\widehat{\alpha}((u \otimes \lambda)(f))\left(\xi \otimes_{\mu} h\right)\right\|^{2}=\int \lambda^{\prime}\left(|f|^{2}\right)(x) \lambda\left(f_{\xi}^{2}\right)(x) d \mu(x),
$$

where $f_{\xi}(\gamma)=\|\xi(\gamma)\|$.

Proof. We first note that

$$
\begin{aligned}
\left\|f \times_{\mu} \xi\right\|^{2} & =\left\|f \otimes_{\mu} \xi\right\|^{2} \\
& =\left(f \otimes_{\mu} \xi \mid f \otimes_{\mu} \xi\right) \\
& =\left(\left(\lambda^{\prime}\left(|f|^{2}\right) \otimes_{\mathscr{L}} 1\right) \xi \mid \xi\right) \\
& =\int \lambda^{\prime}\left(|f|^{2}\right)(x) \lambda\left(f_{\xi}^{2}\right)(x) d \mu(x) .
\end{aligned}
$$

Thus the assertion is equivalent to

$$
\sup _{h \in \mathscr{P}(9)}\left\|\widehat{\alpha}((u \otimes \lambda)(f))\left(\xi \otimes_{\mu} h\right)\right\|^{2}=\left\|f \times_{\mu} \xi\right\|^{2} .
$$

However, thanks to Remark 2.6 and Lemma 3.5 , the quantity on the left-hand side is majorized by the one on the right.

To show the reverse inequality, we introduce a linear map $\zeta$ from $\pi_{r}\left(\mathfrak{B}_{I}\right)$ into $\widetilde{\mathscr{H}}$ given by

$$
\zeta\left(\pi_{r}(h)\right)=\widehat{\alpha}((u \otimes \lambda)(f))\left(\xi \otimes_{\mu} h\right) \quad\left(h \in \mathfrak{B}_{I}\right) .
$$


Since $\pi_{r}$ is one-to-one, the map is well-defined. It is also continuous by Lemma 3.5. So it can be extended to a bounded linear map, denoted by $\zeta$ again, from $A_{r}=$ the uniform closure of $\pi_{r}\left(\mathfrak{B}_{I}\right)$ into $\widetilde{\mathscr{H}}$. If $h, k \in \mathfrak{B}_{I}$, then

$$
\begin{aligned}
\left(1 \otimes_{\mathscr{L}} \pi_{r}(h)\right) \zeta\left(\pi_{r}(k)\right) & =\left(1 \otimes_{\mathscr{L}} \pi_{r}(h)\right) \widehat{\alpha}((u \otimes \lambda)(f))\left(\xi \otimes_{\mu} k\right) \\
& =\widehat{\alpha}((u \otimes \lambda)(f))\left(\xi \otimes_{\mu} k * h\right) \\
& =\zeta\left(\pi_{r}(k * h)\right) \\
& =\zeta\left(\pi_{r}(h) \pi_{r}(k)\right) .
\end{aligned}
$$

Thus continuity of $\zeta$ implies that $\left(1 \otimes_{\mathscr{L}} a\right) \zeta(b)=\zeta(a b)\left(a, b \in A_{r}\right)$. Let $\left\{h_{n}\right\}_{n \geq 1}$ be the sequence that appeared in the proof of Lemma 3.5. We remark that $\left\{\pi_{r}\left(h_{n}\right)\right\}$ is an approximate identity for $A_{r}$. By Lemma 3.5, $\left\{\zeta\left(\pi_{r}\left(h_{n}\right)\right)\right\}$ is bounded; so, by considering its subsequence, we may assume that $\left\{\zeta\left(\pi_{r}\left(h_{n}\right)\right)\right\}$ converges weakly to an element $\zeta_{0} \in \widetilde{\mathscr{H}}$. Then, for any $a \in A_{r}$, we have

$$
\begin{aligned}
\left(\zeta(a) \mid \zeta_{0}\right) & =\lim _{n \rightarrow \infty}\left(\zeta\left(a \pi_{r}\left(h_{n}\right)\right) \mid \zeta_{0}\right) \\
& =\lim _{n \rightarrow \infty}\left(\left(1 \bigotimes_{\mathscr{L}} a\right) \zeta\left(\pi_{r}\left(h_{n}\right)\right) \mid \zeta_{0}\right) \\
& =\left(\left(1 \otimes_{\mathscr{L}} a\right) \zeta_{0} \mid \zeta_{0}\right) .
\end{aligned}
$$

The calculation shows that the functional $a \in A_{r} \mapsto\left(\zeta(a) \mid \zeta_{0}\right)$ extends to a normal positive functional $\varphi_{\zeta}$ on $\mathscr{R}(\mathscr{G})^{\prime}=$ the weak closure of $A_{r}$. Since $\left\{\mathscr{R}(\mathscr{G})^{\prime}\right.$, $\left.L^{2}(\mathscr{G}, v)\right\}$ is a standard representation, there exists a vector $\xi \in L^{2}(\mathscr{G}, v)$ such that $\varphi_{\zeta}=\omega_{\xi}$. For any $h \in \mathfrak{B}_{I}$, we obtain

$$
\begin{aligned}
\|\xi * h\|^{2} & =\omega_{\xi}\left(\pi_{r}(h)^{+} \pi_{r}(h)\right) \\
& =\varphi_{\zeta}\left(\pi_{r}(h)^{+} \pi_{r}(h)\right) \\
& =\left(\zeta\left(\pi_{r}(h)^{+} \pi_{r}(h)\right) \mid \zeta_{0}\right) \\
& =\left(\zeta\left(\pi_{r}(h)\right) \mid\left(1 \otimes_{\mathscr{L}} \pi_{r}(h)\right) \zeta_{0}\right) \\
& =\lim _{n \rightarrow \infty}\left(\zeta\left(\pi_{r}(h)\right) \mid\left(1 \otimes_{\mathscr{Q}} \pi_{r}(h)\right) \zeta\left(\pi_{r}\left(h_{n}\right)\right)\right) \\
& =\lim _{n \rightarrow \infty}\left(\zeta\left(\pi_{r}(h)\right) \mid \zeta\left(\pi_{r}(h) \pi_{r}\left(h_{n}\right)\right)\right) \\
& =\left\|\zeta\left(\pi_{r}(h)\right)\right\|^{2} \\
& =\left\|\widehat{\alpha}((u \otimes \lambda)(f))\left(\xi \otimes_{\mu} h\right)\right\|^{2} .
\end{aligned}
$$

Since $\|\xi\|^{2}=\sup \left\{\|\xi * h\|^{2}: h \in \mathfrak{B}_{I}\right.$ with $\left.\left\|\pi_{r}(h)\right\| \leq 1\right\}$, it follows from the above calculation that

$$
\begin{aligned}
\left\|\zeta_{0}\right\|^{2}=\|\xi\|^{2} & =\sup \left\{\|\xi * h\|^{2}: h \in \mathfrak{B}_{I} \text { with }\left\|\pi_{r}(h)\right\| \leq 1\right\} \\
& =\sup \left\{\left\|\widehat{\alpha}((u \otimes \lambda)(f))\left(\xi \otimes_{\mu} h\right)\right\|^{2}: h \in \mathfrak{B}_{I} \text { with }\left\|\pi_{r}(h)\right\| \leq 1\right\} \\
& \leq \sup _{h \in \mathscr{P}(\mathscr{\varphi})}\left\|\widehat{\alpha}((u \otimes \lambda)(f))\left(\xi \otimes_{\mu} h\right)\right\|^{2} \\
& \leq\left\|f \times_{\mu} \xi\right\|^{2} .
\end{aligned}
$$

Hence it suffices to show $\left\|\zeta_{0}\right\|=\left\|f \times_{\mu} \xi\right\|$. Recall that $\zeta_{0}$ is the weak limit of the 
sequence $\left\{\alpha((u \otimes \lambda)(f))\left(\xi \otimes_{\mu} h_{n}\right)\right\}_{n \geq 1}$. This fact together with (3.9) shows that $\zeta_{0}$ is nothing but $\left(1 \otimes_{\mathscr{L}} \sigma\right) W_{\mathscr{H}}^{+}\left(f \times_{\mu} \xi\right)$. Since both $1 \otimes_{\mathscr{F}} \sigma$ and $W_{\mathscr{H}}^{+}$are unitaries, we obtain the desired identity $\left\|\zeta_{0}\right\|=\left\|f \times_{\mu} \xi\right\|$.

Q.E.D.

Theorem 3.11. The restriction $T_{\alpha}$ of the map $S$ to the crossed product $M \times_{\alpha} \mathscr{G}$ is a faithful normal semifinite operator valued weight of $\mathcal{M} \times_{\alpha} G$ onto $\mathcal{M} \otimes_{\mathscr{L}} \mathbb{C}$.

Proof. Since the restriction of the morphism $\Pi$ to the crossed product is the dual coaction $\widehat{\alpha}$, we have

$$
T_{\alpha}(Y)(\omega)=\left\langle\widehat{\alpha}(Y), \omega *_{\mu} \varphi_{\mathscr{g}}\right\rangle=\sup _{h \in \mathscr{P}(\mathscr{G})}\left\langle\widehat{\alpha}(Y), \omega *_{\mu} \omega_{h}\right\rangle
$$

for any $Y \in\left(\mathcal{M} \times_{\alpha} \mathscr{G}\right)_{+}$and $\omega \in\left(\mathcal{M} \times_{\alpha} \mathscr{G}\right)_{+}^{+}$. Then $T_{\alpha}$ is a normal, homogeneous, additive map from $\left(M \times_{\alpha} \mathscr{G}\right)_{+}$to $\mathscr{E}^{+}\left(M \times_{\alpha} \mathscr{G}\right)$. In view of (3.2), we have

$$
\left(a \bigotimes_{\mathscr{L}} 1\right)^{+} T_{\alpha}(Y)\left(a \otimes_{\mathscr{L}} 1\right)=T_{\alpha}\left(\left(a \otimes_{\mathscr{L}} 1\right)^{+} Y\left(a \bigotimes_{\mathscr{L}} 1\right)\right) \quad\left(Y \in\left(M \times_{\alpha} \mathscr{G}\right)_{+}, a \in \mathcal{M}\right) .
$$

Let us fix an element $Y \in\left(\mathcal{M} \times{ }_{\alpha} \mathscr{G}\right)_{+}$and consider the spectral resolution of $T_{\alpha}(Y)($ see $[\mathrm{H} 2])$ :

$$
T_{\alpha}(Y)=\int_{0}^{\infty} \lambda d e_{\lambda}+\infty \cdot p
$$

From Proposition 3.4, it follows that operators $e_{\lambda}(\lambda \in[0, \infty))$ and $p$ belong to the algebra $\left(M \times_{\alpha} G\right) \cap\left(\mathbb{C} \otimes_{\mathscr{L}} L^{x}(\mathscr{G}, v)\right)^{\prime}$. Thus we have

$$
\left[e_{\lambda}, 1 \otimes_{\mathscr{L}} M(k)\right]=0 \quad\left[p, 1 \bigotimes_{\mathscr{L}} M(k)\right]=0
$$

for any $k \in L^{\infty}(\varphi, v)$. Now we can follow the argument of the proof of Theorem 2.6 of [Y3] in order to conclude that $e_{\lambda}, p$ in fact belong to $M \otimes_{\mathscr{Q}} \mathbb{C}$. Hence $T_{\alpha}(Y)$ lies in $\mathscr{E}^{+}\left(\mathcal{M} \otimes_{\mathscr{Q}} \mathbb{C}\right)$. Thus we have shown that $T_{\alpha}$ is a normal operator valued weight from $M \times_{\alpha} \varphi$ to $M \otimes_{\mathscr{X}} \mathbf{C}$.

Suppose that $T_{\alpha}\left(Y^{+} Y\right)=0$ for some $Y \in M \times_{\alpha} \mathscr{G}$. This means that, for any $\xi \in \widehat{\mathscr{H}}$, we have to have

$$
\begin{aligned}
0 & =T_{\alpha}\left(Y^{+} Y\right)\left(\omega_{\xi}\right) \\
& =\sup _{h \in \mathscr{P}(\mathscr{G})}\left\langle\widehat{\alpha}\left(Y^{+} Y\right), \omega_{\xi}{ }_{\mu} \omega_{h}\right\rangle \\
& =\sup _{h \in \mathscr{P}(\mathscr{G})}\left\|\widehat{\alpha}(Y)\left(\xi \otimes_{\mu} h\right)\right\|^{2} .
\end{aligned}
$$

It follows that $\widehat{\alpha}(Y)\left(\xi \otimes_{\mu} h\right)=0$ for any $\xi \in \widehat{\mathscr{H}}$ and $h \in \mathscr{P}(\mathscr{G})$. Since $\mathscr{P}(\mathscr{G})$ is total in $L^{2}(G, v)$, the operator $\widehat{\alpha}(Y)$ must be 0 , which implies that $Y=0$. Hence $T_{\alpha}$ is faithful.

It remains to show semifiniteness of $T_{\alpha}$. Let $f \in \mathfrak{B}_{I}^{\infty}$. Then

$$
\left\langle\widehat{\alpha}\left((u \otimes \lambda)\left(f^{\#} * f\right)\right), \omega_{\xi \otimes_{u} h}\right\rangle=\left\|\widehat{\alpha}((u \otimes \lambda)(f))\left(\xi \otimes_{\mu} h\right)\right\|^{2}
$$

for any $h \in \mathscr{P}(\mathscr{G})$ and $\xi \in \widehat{\mathscr{H}}$. By Lemma 3.10, we have 


$$
\begin{aligned}
T_{\alpha}\left((u \otimes \lambda)\left(f^{\#} * f\right)\right)\left(\omega_{\xi}\right) & =\sup _{h \in \mathscr{P}(\mathscr{\xi})}\left\|\widehat{\alpha}((u \otimes \lambda)(f))\left(\xi \otimes_{\mu} h\right)\right\|^{2} \\
& =\int \lambda^{\prime}\left(|f|^{2}\right)(x) \lambda\left(f_{\xi}^{2}\right)(x) d \mu(x) \\
& =\left\langle\lambda^{\prime}\left(|f|^{2}\right) \otimes_{\mathscr{F}^{\prime}} 1, \omega_{\xi}\right\rangle .
\end{aligned}
$$

This shows that $T_{\alpha}\left((u \otimes \lambda)\left(f^{\#} * f\right)\right)=\lambda^{\prime}\left(|f|^{2}\right) \otimes_{\mathscr{L}} 1$. It follows that the operator $(u \otimes \lambda)(f)\left(f \in \mathfrak{B}_{I}^{\infty}\right)$ belongs to the left ideal $\mathrm{n}_{T_{n}}$. Accordingly, the $*$-algebra $(u \otimes \lambda)\left(\mathfrak{B}_{I}^{\infty}\right)$ is contained in $\mathrm{n}_{T_{\mathrm{a}}}$. Since $\mathrm{n}_{T_{\mathrm{a}}}$ is a two-sided module over $M \otimes_{\mathscr{L}} \mathbf{C}$, it also contains the *-algebra generated by $\mathcal{M} \otimes_{\mathscr{L}} \mathbf{C}$ and $(u \otimes \lambda)\left(\mathfrak{B}_{I}^{\infty}\right)$, which is $\sigma$-weakly dense in $\mathcal{M} \times_{\alpha} \mathscr{G}$. Therefore, the operator valued weight $T_{\alpha}$ is semifinite.

Q.E.D.

\section{§4. Construction of Dual Weights}

In [Y2], we showed that, to each faithful normal positive functional $\varphi$ on $\mathcal{M}$, there corresponds a faithful normal semifinite weight $\widetilde{\varphi}$ on the crossed product $M \times_{\alpha} \mathscr{G}$, called the dual weight of $\varphi$. Meanwhile, we may define, for a given $\varphi$, a faithful normal semifinite weight $\widehat{\varphi}$ on $\mathcal{M} \times_{\alpha} \mathscr{G}$ by $\widehat{\varphi}=\varphi \circ I_{\alpha}^{-1} \circ T_{\alpha}$, using the operator valued weight $T_{\alpha}$, where $I_{\alpha}$ is the canonical injection of $\mu$ into $\mathcal{M} \otimes_{\mathscr{L}} \mathbf{C}$. Our aim in the following discussion is to show that the dual weight $\tilde{\varphi}$ coincides with $\widehat{\varphi}$. Thus the dual weight construction introduced in [Y2] can be extended by making use of the operator valued weight $T_{\alpha}$, as in the case of group actions.

Let $\left(\mathscr{G},\{\mathcal{M}(x)\},\left\{\alpha_{\gamma}\right\}\right)$ be an action of $\mathscr{G}$. In what follows, we keep all the relevant notations introduced in the preceding sections. Let us fix a faithful normal positive functional $\varphi$ on $\mathcal{M}$. We consider a direct intergal decomposition $\varphi=\int_{X}^{\oplus} \varphi_{x} d \mu(x)$ relative to $\mu=\int_{X}^{\oplus} \mu(x) d \mu(x)$ (see [Su]). We may assume that each $\varphi_{x}$ is a faithful normal positive functional on $\mathcal{M}(x)$; so we may identify $\mathscr{H}_{\varphi_{1}}$ (resp. $\mathscr{H}$ ), the Hilbert space obtained by GNS construction from $\varphi_{x}$ (resp. $\varphi$ ), with the canonical $L^{2}$-space $\mathscr{H}(x)$ (resp. $\mathscr{H}$ ) of $\mathcal{M}(x)$ (resp. $\mathcal{M}$ ). In [Y2], we constructed, from the given system, a left Hilbert algebra $\widetilde{\mathfrak{T}}_{\varphi}$ whose left von Neumann algebra coincides with the crossed product. Let $\widetilde{\Delta}_{\varphi}$ be the modular operator associated with $\widetilde{\mathfrak{T}}_{\varphi}$. (See Lemma 2.6 of [Y2] for $\widetilde{\Delta}_{\varphi}$ ). Since $\widetilde{\Delta}_{\varphi}^{i t}$ commutes with the canonical $\mathscr{L}$-action on $\widehat{\mathscr{H}}, \widetilde{\Delta}_{\varphi}^{i t} \bigotimes_{\mathscr{L}} 1$ makes sense on $\widetilde{\mathscr{H}}$. We set $\sigma_{t}^{\widetilde{\varphi}} \otimes_{{ }_{\Psi}} 1=\operatorname{Ad}\left(\widetilde{\Delta}_{\varphi}^{i t} \otimes_{\mathscr{L}} 1\right)$.

Proposition 4.1. With the notations as above, we have

$$
\widehat{\alpha} \circ \sigma_{t}^{\widetilde{\varphi}}=\left(\sigma_{t}^{\widetilde{\varphi}} \otimes_{\mathscr{L}} 1\right) \circ \widehat{\alpha} \quad(t \in \mathbf{R}) .
$$

Proof. First we note that $\left[\widetilde{\Delta}_{\varphi}^{i t}, 1 \bigotimes_{\mathscr{L}} M(h \circ s)\right]=0$ for any $t \in \mathbf{R}$ and $h \in \mathscr{L}$. This fact can be easily verified due to Lemma 2.6 of [Y2]. Thus $\widetilde{\Delta}_{\varphi}^{i t} \widehat{\otimes}_{\mathscr{L}} 1$ makes sense on $\widehat{\mathscr{H}}_{\mathscr{L}} \otimes_{\mu \mathscr{L}} L^{2}(\mathscr{G}, v)$. (Recall the notation $\widehat{\otimes}_{\mathscr{L}}$ introduced in $\left.\$ 3\right)$. For any $\xi \in \widehat{\mathscr{H}}$ and $f \in \mathfrak{B}_{I}$, consider the vector $\xi \widehat{\otimes}_{\mu} f$ in $\widehat{\mathscr{H}}_{\mathscr{L}} \otimes_{\mu \mathscr{L}} L^{2}(\mathscr{G}, v)$. (See $\S 3$ again 
for $\widehat{\bigotimes}_{\mu}$ ). Then we have

$$
\begin{aligned}
\left\{W_{\mathscr{H}}^{+} V_{\mathscr{H}}\left(\widetilde{\Delta}_{\varphi}^{i t} \widehat{\otimes}_{\mathscr{L}} 1\right)\left(\xi \widehat{\otimes}_{\mu} f\right)\right\}\left(\gamma_{1}, \gamma_{2}\right) & =f\left(\gamma_{1}^{-1} \gamma_{2}\right)\left\{\widetilde{\Delta}_{\varphi}^{i t} \xi\right\}\left(\gamma_{1}\right) \\
& =\left\{\left(\widetilde{\Delta}_{\varphi}^{i t} \otimes_{\mathscr{L}} 1\right) W_{\mathscr{H}}^{i} V_{\mathscr{H}}\left(\xi \widehat{\otimes}_{\mu} f\right)\right\}\left(\gamma_{1}, \gamma_{2}\right) .
\end{aligned}
$$

The above equalities are due to the fact that $\left\{W_{\mathscr{H}}^{+} V_{\mathscr{H}}\left(\xi \widehat{\otimes}_{\mu} f\right)\right\}\left(\gamma_{1}, \gamma_{2}\right)=f\left(\gamma_{1}^{-1} \gamma_{2}\right)$ $\xi\left(\gamma_{1}\right)$. Hence we have shown that $W_{\mathscr{H}}^{+} V_{\mathscr{H}}\left(\widetilde{\Delta}_{\varphi}^{i t} \widehat{\otimes}_{\mathscr{L}} 1\right)=\left(\widetilde{\Delta}_{\varphi}^{i t} \otimes_{\mathscr{H}} 1\right) W_{\mathscr{H}}^{+} V_{\mathscr{H}}$. From this, it follows that, for any $Y \in M \times_{\alpha} \mathscr{G}$, we have

$$
\begin{aligned}
\widehat{\alpha}^{\circ} \sigma_{t}^{\widetilde{\varphi}}(Y) & =W_{\mathscr{H}}^{+} V_{\mathscr{H}}\left(\sigma_{t}^{\widetilde{\varphi}}(Y) \widehat{\otimes}_{\mathscr{L}} 1\right) V_{\mathscr{H}}^{+} W_{\mathscr{H}} \\
& =W_{\mathscr{H}}^{+} V_{\mathscr{H}}\left(\left(\widetilde{\Delta}_{\varphi}^{i t} \widehat{\bigotimes}_{\mathscr{L}} 1\right)\left(Y \widehat{\bigotimes}_{\mathscr{L}} 1\right)\left(\widetilde{\Delta}_{\varphi}^{-i t} \widehat{\bigotimes}_{\mathscr{L}} 1\right) V_{\mathscr{H}}^{+} W_{\mathscr{H}}\right. \\
& =\left(\widetilde{\Delta}_{\varphi}^{i t} \bigotimes_{\mathscr{H}} 1\right) W_{\mathscr{H}}^{+} V_{\mathscr{H}}\left(Y \widehat{\bigotimes}_{\mathscr{L}} 1\right) V_{\mathscr{H}}^{+} W_{\mathscr{H}}\left(\widetilde{\Delta}_{\varphi}^{-i t} \bigotimes_{\mathscr{L}} 1\right) \\
& =\left(\sigma_{t}^{\widetilde{\varphi}} \bigotimes_{\mathscr{H}} 1\right) \circ \widehat{\alpha}(Y) .
\end{aligned}
$$

Therefore, we obtain the desired identity.

Q.E.D.

Lemma 4.2. The weight $\widehat{\varphi}$ is $\sigma^{\widetilde{\varphi}}$-invariant. Namely,

$$
\widehat{\varphi} \circ \sigma_{t}^{\widetilde{\varphi}}=\widehat{\varphi} \quad(t \in \mathbf{R}) .
$$

Proof. Let $\omega \in\left(\mathcal{M} \times{ }_{\alpha} \mathscr{G}\right)_{*}^{+}$and $Y \in\left(\mathcal{M} \times{ }_{\alpha} \mathscr{G}\right)_{+}$. Then $\omega$ is of the form $\omega=\omega_{\xi}$ for some $\xi \in \widehat{\mathscr{H}}$. It follows from the definition of $T_{\alpha}$ and Proposition 4.1 that

$$
\begin{aligned}
T_{\alpha}\left(\sigma_{t}^{\widetilde{\varphi}}(Y)\right)(\omega) & =\sup _{h \in \mathscr{P}(\mathscr{G})}\left\langle\widehat{\alpha}\left(\sigma_{t}^{\widetilde{\varphi}}(Y)\right), \omega_{\xi \otimes_{u} h}\right\rangle \\
& =\sup _{h \in \mathscr{P}(\mathscr{G})}\left\langle\left(\sigma_{t}^{\widetilde{\varphi}} \otimes_{\mathscr{Z}} 1\right) \circ \widehat{\alpha}(Y), \omega_{\xi \otimes_{u} h}\right\rangle \\
& =\sup _{h \in \mathscr{P}(\mathscr{G})}\left\langle\widehat{\alpha}(Y), \omega_{\widetilde{\Delta}_{q}^{-\prime} \xi \otimes_{\mu} h}\right\rangle \\
& =T_{\alpha}(Y)\left(\omega_{\widetilde{\Delta}_{q}^{-\prime} \xi}\right) \\
& =T_{\alpha}(Y)\left(\omega^{\circ} \sigma_{t}^{\widetilde{\varphi}}\right) \\
& =\sigma_{t}^{\widetilde{\varphi}} \circ T_{\alpha}(Y)(\omega) .
\end{aligned}
$$

This shows that $T_{\alpha}\left(\sigma_{t}^{\widetilde{\varphi}}(Y)\right)=\sigma_{t}^{\widetilde{\varphi}} \circ T_{\alpha}(Y)$. Since $T_{\alpha}(Y)$ lies in $\mathscr{E}^{+}\left(M \otimes_{\mathscr{E}} \mathbf{C}\right)$ and since $\sigma_{t}^{\widetilde{\varphi}}\left(a \otimes_{\mathscr{L}} 1\right)=\sigma_{t}^{\varphi}(a) \bigotimes_{\mathscr{L}} 1$ for any $a \in M$ by Lemma 2.16 of [Y2], we have that $\sigma_{t}^{\widetilde{\varphi}} \circ T_{\alpha}(Y)=I_{\alpha} \circ \sigma_{t}^{\varphi} \circ I_{\alpha}^{-1} \circ T_{\alpha}(Y)$. Consequently, we obtain $T_{\alpha}\left(\sigma_{t}^{\widetilde{\varphi}}(Y)\right)=I_{\alpha} \circ$ $\sigma_{t}^{\varphi} \circ I_{\alpha}^{-1} \circ T_{\alpha}(Y)$. From this, it follows that

$$
\begin{aligned}
\widehat{\varphi} \circ \sigma_{t}^{\widetilde{\varphi}}(Y) & =\varphi \circ I_{\alpha}^{-1} \circ T_{\alpha}\left(\sigma_{t}^{\widetilde{\varphi}}(Y)\right) \\
& =\varphi \circ I_{\alpha}^{-1} \circ I_{\alpha} \circ \sigma_{t}^{\varphi} \circ I_{\alpha}^{-1} \circ T_{\alpha}(Y) \\
& =\varphi \circ \sigma_{t}^{\varphi} \circ I_{\alpha}^{-1} \circ T_{\alpha}(Y) \\
& =\varphi \circ I_{\alpha}^{-1} \circ T_{\alpha}(Y) \\
& =\widehat{\varphi}(Y) .
\end{aligned}
$$


This completes the proof.

Q.E.D.

Lemma 4.3. Let $a \in \mathscr{F}_{\varphi}^{\infty} \cap\left(\mathscr{F}_{\varphi}^{\infty}\right)^{\#}$ and $\xi \in \widehat{\mathscr{H}}$. Then the function $a \times_{\mu} \xi$ given by $\left(a \times_{\mu} \xi\right)\left(\gamma_{1}, \gamma_{2}\right)=a\left(\gamma_{1}\right) u\left(\gamma_{1}\right) \xi\left(\gamma_{1}^{-1} \gamma_{2}\right)\left(\left(\gamma_{1}, \gamma_{2}\right) \in \mathscr{H}^{(2)}\right)$ belongs to $\widetilde{\mathscr{H}}$. Moreover, we have

$$
\left\|\widehat{\alpha}\left(\Phi\left(A_{a}\right)\right)\left(\xi \otimes_{\mu} h\right)\right\| \leq\left\|\pi_{r}(h)\right\|\left\|a \times_{\mu} \xi\right\|
$$

for any right bounded vector $h \in D\left(\mathscr{L} L^{2}(\mathscr{G}, v), \mu\right)$.

Proof. Define functions $f_{a}$ and $f_{\xi}$ by $f_{a}(\gamma)=\|a(\gamma)\|, f_{\xi}(\gamma)=\|\xi(\gamma)\|$. Then

$$
\begin{aligned}
\int\left\|\left(a \times_{\mu} \xi\right)\left(\gamma_{1}, \gamma_{2}\right)\right\|^{2} d v_{1}\left(\gamma_{1}, \gamma_{2}\right) & \leq \iint f_{a}\left(\gamma_{1}\right)^{2} f_{\xi}\left(\gamma_{1}^{-1} \gamma_{2}\right)^{2} d \lambda^{r\left(\gamma_{1}\right)}\left(\gamma_{2}\right) d v\left(\gamma_{1}\right) \\
& =\iint f_{a}\left(\gamma_{1}\right)^{2} f_{\xi}\left(\gamma_{2}\right)^{2} d \lambda^{s\left(\gamma_{1}\right)}\left(\gamma_{2}\right) \delta\left(\gamma_{1}\right) d \lambda_{x}\left(\gamma_{1}\right) d \mu(x) \\
& =\int\left(\int f_{a}\left(\gamma_{1}\right)^{2} \delta\left(\gamma_{1}\right) d \lambda_{r\left(\gamma_{2}\right)}\left(\gamma_{1}\right)\right) f_{\xi}\left(\gamma_{2}\right)^{2} d v\left(\gamma_{2}\right) \\
& \leq\left\|\lambda^{\prime}\left(f_{a}^{2}\right)\right\|_{\infty}\|\xi\|^{2}<\infty .
\end{aligned}
$$

Thus $a \times{ }_{\mu} \xi$ belongs to $\widetilde{\mathscr{H}}$.

The idea of proving the second assertion is the same as that of Lemma 3.5. Let $h \in D\left({ }^{2} L^{2}(\varphi, v), \mu\right)$ be right bounded and $k \in L^{2}(G, v)$. Suppose that $\eta$ is in $\widehat{\mathscr{H}}$ such that $f_{\eta} \in \mathfrak{B}_{I}\left(f_{\eta}(\gamma)=\|\eta(\gamma)\|\right)$. Then it follows from Fubini's theorem that

$$
\left(\widehat{\alpha}\left(\Phi\left(A_{a}\right)\right)\left(\xi \otimes_{\mu} h\right) \mid \eta \otimes_{\mu} k\right)=\int(p * h)(\gamma) \overline{k(\gamma)} d v(\gamma)
$$

where $p(\gamma)=\int\left(a(\gamma) u(\gamma) \xi\left(\gamma^{-1} \gamma_{1}\right) \mid \eta\left(\gamma_{1}\right) d \lambda^{r(\gamma)}\left(\gamma_{1}\right)\right.$. We assert that the function $p$ belongs to $L^{2}(\xi, v)$. In fact, it is easy to see that $|p| \leq f_{a} \cdot\left(f_{\eta} * f_{\xi}^{b}\right)$. By the argument similar to the one in Lemma 3.5 where we showed that $f \cdot p \in L^{2}(\mathscr{G}, v)$, we can prove that $p \in L^{2}(\varphi, v)$. Hence $(4.4)$ is equivalent to

$$
\left(\widehat{\alpha}\left(\Phi\left(A_{a}\right)\right)\left(\xi \otimes_{\mu} h\right) \mid \eta \otimes_{\mu} k\right)=\left(\pi_{r}(h) p \mid k\right) .
$$

It is a straightforward calculation to check

$$
\left(\left(a \otimes_{\mu} \xi\right) \mid \eta \otimes_{\mu} k\right)=(p \mid k) .
$$

Once we have obtained these identities (4.5) and (4.6), we can easily show by following the discussion in Lemma 3.5 that

$$
\widehat{\alpha}\left(\Phi\left(A_{a}\right)\right)\left(\xi \otimes_{\mu} h\right)=\left(1 \bigotimes_{\mathscr{Z}} \pi_{r}(h)\right)\left(a \times_{\mu} \xi\right) .
$$

From this, the second assertion follows.

Q.E.D.

Corollary 4.7. Let $a$ and $\xi$ be as in Lemma 4.3. Then 


$$
\sup _{h \in \mathscr{P}(\mathscr{G})}\left\|\widehat{\alpha}\left(\Phi\left(A_{a}\right)\right)\left(\xi \otimes_{\mu} h\right)\right\|^{2}=\left\|a \times_{\mu} \xi\right\|^{2} .
$$

The idea of the proof is quite the same as that of Lemma 3.6. Hence we leave the proof to readers as exercise.

Let $q$ be a nonnegative Borel function on $\mathscr{G}$ such that $\int q(\gamma) d \lambda^{x}(\gamma)=1$ for all $x \in X$. The existence of such a function is guaranteed by Lemma 3 of [C1]. Let us take increasing Borel subsets $\left\{X_{n}\right\}_{n \geq 1}$ of $X$ with $X=\cup_{n=1}^{\infty} X_{n}, \mu\left(X_{n}\right)<\infty$. We define $q_{n}(n \geq 1)$ by $q_{n}(\gamma)=1_{X_{n}}(r(\gamma)) \sqrt{q(\gamma)}$. Then $q_{n} \in L^{2}(G, v)$. In fact, $q_{n}$ belongs to $D\left(L_{L} L^{2}(\mathscr{G}, v), \mu\right)$. Let $\omega \in \mathcal{M}_{+}^{+}$. Since $\{\mathcal{M}, \mathscr{H}\}$ is standard, there

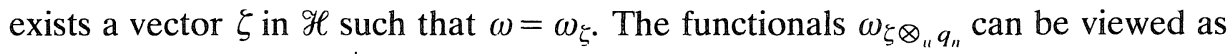
elements in $\left(\mathcal{M} \otimes_{\mathscr{L}} \mathbb{C}\right)_{+}^{+}$. Then we have the following lemma.

Lemma 4.8. With the notations as above, we have

$$
\omega \circ I_{\alpha}^{-1}=\sup _{n \geq 1} \omega_{\zeta \otimes_{u}} q_{n}
$$

Proof. Since $\lambda\left(\left|q_{n}\right|^{2}\right)=1_{X_{n}} \leq 1_{X_{m}}=\lambda\left(\left|q_{m}\right|^{2}\right)$ for any $n \leq m$, it follows that

$$
\begin{aligned}
\omega_{\zeta \otimes_{1} q_{n}}\left(a^{+} a \otimes_{\mathscr{X}} 1\right) & =\left\|a \zeta \otimes_{\mu} q_{n}\right\|^{2} \\
& =\left(\lambda\left(\left|q_{n}\right|^{2} a \zeta \mid a \zeta\right)\right. \\
& \leq\left(\lambda\left(\left|q_{m}\right|^{2} a \zeta \mid a \zeta\right)\right. \\
& =\omega_{\zeta \otimes_{u} q_{m}}\left(a^{+} a \bigotimes_{\mathscr{X}} 1\right)
\end{aligned}
$$

for any $a \in \mathcal{M}$ and $n \leq m$. Thus $\omega_{\zeta \otimes_{\mu} q_{n}} \leq \omega_{\zeta \otimes_{\mu} q_{m}}(n \leq m)$ as functionals in $\left(M \otimes_{\mathscr{L}} \mathbb{C}\right)_{+}^{+}$. Moreover,

$$
\begin{aligned}
\sup _{n \geq 1} \omega_{\zeta \otimes_{n} q_{n}}\left(a^{+} a \otimes_{\mathscr{L}} 1\right) & =\sup _{n \geq 1}\left(\lambda\left(\left|q_{n}\right|^{2}\right) a \xi \mid a \zeta\right) \\
& =\sup _{n \geq 1}\left\|1_{X_{n}} a \zeta\right\|^{2} \\
& =\|a \zeta\|^{2}=\omega \circ I_{\alpha}^{-1}\left(a^{+} a \bigotimes_{\mathscr{L}} 1\right)
\end{aligned}
$$

Therefore, we get the desired indentity.

Q.E.D.

Theorem 4.9. The dual weight $\tilde{\varphi}$ coincides with $\widehat{\varphi}=\varphi \circ I_{\alpha}^{-1} \circ T_{\alpha}$.

Proof. We write $\varphi$ as $\varphi=\omega_{\zeta}$ for some vector $\zeta \in \mathscr{H}$. We consider the decomposition $\zeta=\int_{X}^{\oplus} \zeta_{x} d \mu(x)$ relative to the direct integral $\mathscr{H}=\int_{X}^{\oplus} \mathscr{H}(x) d \mu(x)$. Then we have that $\omega_{\zeta_{1}}=\varphi_{x}$ for a.e. $x$. We take the functions $\left\{q_{n}\right\}$ introduced as above. Let $a \in \mathscr{F}_{\varphi}^{\infty} \cap\left(\mathscr{F}_{\varphi}^{\infty}\right)^{\#}$. Then, by Corollary 4.7, we have

$$
\begin{aligned}
& T_{\alpha}\left(\Phi\left(A_{a}\right)^{+} \Phi\left(A_{a}\right)\right)\left(\omega_{\zeta \otimes_{n} q_{n}}\right) \\
& =\sup _{h \in \mathscr{P}(\mathscr{G})}\left\langle\widehat{\alpha}\left(\Phi\left(A_{a}\right)^{+} \Phi\left(A_{a}\right)\right), \omega_{\zeta \otimes_{1} q_{n} \otimes_{1} h}\right\rangle \\
& =\sup _{h \in \mathscr{P}(\mathscr{G})} \| \widehat{\alpha}\left(\Phi\left(A_{a}\right)\left(\zeta \otimes_{\mu} q_{n} \otimes_{\mu} h\right) \|^{2}\right.
\end{aligned}
$$




$$
\begin{aligned}
& =\left\|a \times_{\mu}\left(\zeta \otimes_{\mu} q_{n}\right)\right\|^{2} \\
& =\iint\left|q_{n}\left(\gamma_{1}^{-1} \gamma_{2}\right)\right|^{2}\left\|a\left(\gamma_{1}\right) u\left(\gamma_{1}\right) \zeta_{s\left(\gamma_{1}\right)}\right\|^{2} d \lambda^{r\left(\gamma_{1}\right)}\left(\gamma_{2}\right) d v\left(\gamma_{1}\right) \\
& =\int\left\|a\left(\gamma_{1}\right) u\left(\gamma_{1}\right) \zeta_{s(\gamma)}\right\|^{2}\left(\int\left|q_{n}\left(\gamma_{1}^{-1} \gamma_{2}\right)\right|^{2} d \lambda^{r\left(\gamma_{1}\right)}\left(\gamma_{2}\right)\right) d v\left(\gamma_{1}\right) \\
& =\int\left\|a\left(\gamma_{1}\right) u\left(\gamma_{1}\right) \xi_{s(\gamma)}\right\|^{2}\left(\int\left|q_{n}\left(\gamma_{2}\right)\right|^{2} d \lambda^{s\left(\gamma_{1}\right)}\left(\gamma_{2}\right)\right) d v\left(\gamma_{1}\right) \\
& =\int \varphi_{s\left(\gamma_{1}\right)^{\circ}} \alpha_{\gamma_{1}}^{-1}\left(a\left(\gamma_{1}\right)^{+} a\left(\gamma_{1}\right)\right) 1_{X_{n}}\left(s\left(\gamma_{1}\right)\right) d v\left(\gamma_{1}\right) \text {. }
\end{aligned}
$$

Thus it follows from Lemma 4.8 and Lebesgue dominated convergence theorem that we have

$$
\begin{aligned}
& \varphi\left(\Phi\left(A_{a}\right)^{4} \Phi\left(A_{a}\right)\right)=T_{\alpha}\left(\Phi\left(A_{a}\right)^{4} \Phi\left(A_{\alpha}\right)\right)\left(\varphi \circ I_{\alpha}^{-1}\right) \\
& =\sup _{n \geq 1} T_{\alpha}\left(\Phi\left(A_{a}\right)^{+} \Phi\left(A_{a}\right)\right)\left(\omega_{\zeta \otimes_{n} q_{n}}\right)
\end{aligned}
$$

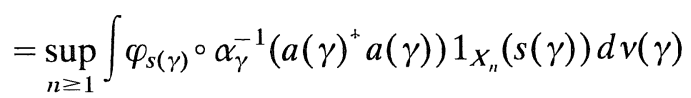

$$
\begin{aligned}
& =\int \varphi_{s(\gamma)}{ }^{\circ} \alpha_{\gamma}^{-1}\left(a(\gamma)^{+} a(\gamma)\right) d v(\gamma) .
\end{aligned}
$$

Meanwhile, $\widetilde{\varphi}\left(\Phi\left(A_{a}\right)^{4} \Phi\left(A_{a}\right)\right)$ is, by definition, equal to

$$
\begin{aligned}
\widetilde{\varphi}\left(\Phi\left(A_{a}\right)^{\prime} \Phi\left(A_{a}\right)\right) & =\left\|\Lambda_{\varphi}(a)\right\|^{2} \\
& =\int\left\|U_{\varphi_{((\gamma)}, \varphi_{s(\nu)}{ }^{\circ} \alpha_{\gamma}^{-1}} \eta_{\varphi_{s(\nu)}{ }^{\circ} \alpha_{\gamma}^{-1}}(a(\gamma))\right\|^{2} d v(\gamma) \\
& =\int\left\|\eta_{\varphi_{s(\nu)} \alpha^{-1}}(a(\gamma))\right\|^{2} d v(\gamma) \\
& =\int \varphi_{s(\gamma)}{ }^{\circ} \alpha_{\gamma}^{-1}\left(a(\gamma)^{+} a(\gamma)\right) d v(\gamma) .
\end{aligned}
$$

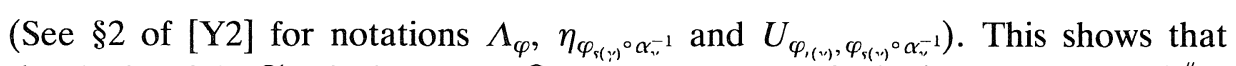
the dual weight $\widetilde{\varphi}$ coincides with $\widehat{\varphi}$ on the $*$-algebra $\left\{\Phi\left(A_{a}\right): a \in \mathscr{F}_{\varphi}^{\infty} \cap\left(\mathscr{F}_{\varphi}^{\infty}\right)^{\#}\right\}$,

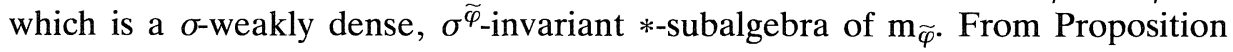
5.9 of $[\mathrm{P} \& \mathrm{~T}]$ and Lemma 4.2 , it follows that $\widetilde{\varphi}=\widehat{\varphi}$.

Q.E.D.

Thanks to Theorem 4.9, the following definition is consistent with the one given in [Y2].

Definition 4.10. For any faithful normal semifinite weight $\varphi$ on $\mathcal{M}$, the equation

$$
\widehat{\varphi}=\varphi \circ I_{\alpha}^{-1} \circ T_{\alpha}
$$

defines a faithful normal semifinite weight on the crossed product $\mathcal{M} \times_{\alpha} \mathscr{G}$. We 
call it the dual weight of $\varphi$.

\section{References}

[C1] Connes, A., Sur la théorie non commutative de l'intégration, Lecture Notes in Math., Springer-Verlag, Berlin, Heidelberg, New York, 725 (1979), 19-143.

[C2] On the spatial theory of von Neumann algebras, J. Functional Analysis, 35 (1980), 153-164.

[D] Digernes, T., Duality for weights on covariant systems and its applications, Dissertation at UCLA, 1975.

[E\&S] Enock, E. and Schwartz, J.M., Une nouvelle construction du poids dual sur le produit criosé d'une algebre de von Neumann par un groupe localement compact, C.R. Acad.Sc. Paris, 282 (1976), Série A, 415-418.

[H1] Haagerup, U., The standard form of von Neumann algebras, Math. Scan., 37 (1975), $271-283$

[H2] Operator valued weights in von Neumann algebras I, J. Functional Analysis, 32 (1979), 175-206.

[H3] Operator valued weights in von Neumann algebras II, J. Functional Analysis, 33 (1979), 339-361.

[H4] On the dual weight for crossed products of von Neumann algebras II, Math. Scan., 43 (1978), 119-140.

[Ha] Hahn, P., The regular representations of measured groupoids, Trans. Amer. Math. Soc., 242 (1978), 34-72.

[K] Kosaki, H., Canonical $L^{p}$-spaces associated with arbitrary abstract von Neumann algebras. Dissertation at UCLA, 1980.

[N\&T] Nakagami, Y. and Takesaki, M., Duality for crossed products of von Neumann algebras, Lecture Notes in Math., Springer-Verlag, Berlin, Heidelberg, New York, 731 (1979).

[P\&T] Pedersen, G.K. and Takesaki, M., The Radon-Nikodym theorem for von Neumann algebras, Acta Math., 130 (1975), 53-87.

[R] Ramsay, A., Virtual group and group actions, Adv. in Math., 6 (1971), 253-322.

[S1] Sauvageot, J.L., Produits tensoriels de Z-modules, Publ. Univ. P. \& M. Curie, 23 (1980).

[S2] _ _ Produits tensoriels de Z-modules et application, Lecture Notes in Math., SpringerVerlag, Berlin, Heidelberg, New York, 1132 (1983), 468-485.

[S3] Sur le produit tensoriel relatif d'espace de Hilbert, J. Operator Theory, 9 (1983), $237-252$.

[Su] Sutherland, C., The direct integral theory of weights, and the Plancherel formula, Dissertation at UCLA, 1973.

[T1] Takesaki, M., Tomita's theory of modular Hilbert algebras and its application, Lecture Notes in Math., Springer-Verlag, Berlin, Heidelberg, New York, 128 (1970).

[T2] Duality for crossed products and the structure of von Neumann algebras of type III, Acta Math., 131 (1973), 249-310.

[T3] Theory of operator algebras I, Springer-Verlag, New York, Heidelberg, Berlin, 1979.

[T4] The structure of operator algebras, Iwanami-shoten, Tokyo, 1983, (in Japanese).

[Y1] Yamanouchi, T., Duality for actions and coactions of measured groupoids on von Neumann algebras, to appear in Memoirs Amer. Math. Soc.

[Y2] Crossed products by groupoid actions and their smooth flows of weights, Publ. RIMS, Kyoto Univ., 28 (1992), 535-576.

[Y3] Fixed point algebras of groupoid actions and coactions, In preparation. 